\title{
Netzwerkbasierte Betrachtung von ko-konstruktiven Interaktionsprozessen im Unterricht - Ein Ansatz zur Beschreibung und Analyse von Angebot und Nutzung
}

\author{
Bianka Troll $(\mathbb{D} \cdot$ Cathleen Heil $(\mathbb{D}) \cdot$ Marcus Pietsch $(\mathbb{D} \cdot$ Michael Besser $(\mathrm{D}$
}

Eingegangen: 19. Februar 2021 / Überarbeitet: 18. Januar 2022 / Angenommen: 2. Februar 2022 / Online publiziert: 25. Februar 2022

(C) Der/die Autor(en) 2022

Zusammenfassung Unterricht stellt ein interaktives Geschehen dar. Die verbale Beteiligung der Schüler*innen an der Interaktion im Unterricht kann dabei als Indikator für die Nutzung von Lernangeboten, aber gleichzeitig auch als potenzielles Lernangebot für andere an der Interaktion beteiligte Schüler*innen interpretiert werden. Der Artikel stellt eine netzwerkbasierte Betrachtung von ko-konstruktiven Interaktionsprozessen vor, um sich der Beschreibung und Analyse von Angebots-Nutzungs-Prozessen im Unterricht anzunähern. Netzwerkbasierte Betrachtungen liefern dabei nicht nur geeignete Visualisierungen, um Interaktionsprozesse übersichtlich darzustellen, sondern auch auf quantitativen Verfahren beruhende Ansatzpunkte für die Analyse von videobasierten Daten. Datengrundlage bildet eine schüler*innenzentriert videografierte Gruppenarbeitsphase (fünf Gruppen á 4 Schüler*innen) im Deutschunterricht einer dritten Klasse. Zur Analyse der dynamischen Interaktionsprozesse wurden die gerichteten Redebeiträge (wer spricht zu wem) der Schüler*innen sowie der Lehrkraft im zeitlichen Verlauf bezüglich ihrer Art (aufgabenbezogen prozessorientiert, aufgabenbezogen ergebnisorientiert, nicht-aufgabenbezogen) von vier geschulten Kodierer*innen kodiert. Die Analysen zeigen, dass sich der methodische Ansatz der Netzwerkanalyse eignet, um abzubilden, wer wie stark an der Bearbeitung einer Lernaufgabe beteiligt ist und wer konkret ein Lernangebot erhält. Zudem kann aufgezeigt werden, wer gemeinsam ko-konstruktiv an der Lösungsfindung beteiligt ist und wie sich der Interaktionsprozesse über die

\footnotetext{
Bianka Troll $(\bowtie) \cdot$ Marcus Pietsch

Institut für Bildungswissenschaft, Leuphana Universität Lüneburg, Lüneburg, Deutschland

E-Mail: bianka.troll@posteo.de

Marcus Pietsch

E-Mail: marcus.pietsch@leuphana.de

Cathleen Heil · Michael Besser

Institut für Mathematik und ihre Didaktik, Leuphana Universität Lüneburg, Lüneburg, Deutschland

E-Mail: michael.besser@leuphana.de
} 
Zeit entwickelt. Die Ergebnisse werden hinsichtlich ihres Mehrwerts und möglicher Anschlussstudien kritisch diskutiert.

Schlüsselwörter Angebots-Nutzungs-Modell · Netzwerkanalyse · Interaktionsprozesse $\cdot$ Unterricht

\title{
A network-based view of co-constructive interaction processes in classrooms-An approach to the description and analysis of opportunity and use
}

\begin{abstract}
Teaching is an interactive process. The verbal participation of students in classroom interactions can be interpreted not only as an indicator of their use of learning opportunities, but also as a potential learning opportunity for other students involved in the interaction process. This article introduces a network-based view of co-constructive interaction processes that describes and analyzes opportunity-use processes in the classroom. The network-based approach enables us to visualize interaction processes while also offering a starting point for the quantitative analysis of video-based data. The present study is based on data gathered from a videotaped student-centered group work session (five groups of four students each) in a third grade German class. To analyze the dynamic interaction processes, the direct contributions (who spoke to whom) of the students and the teacher were coded over time with regard to their type (task-related process-oriented, task-related result-oriented, or non-task-related) by four trained coders. The analyses confirm that network analysis is a valuable methodological approach for examining who engages (and to what extent) in the learning task and who receives verbal learning opportunities. In addition, it allows for an investigation of who is co-constructively involved in the solution process and how the interaction process develops over time. The results are critically discussed with regard to their added value and possible follow-up studies.
\end{abstract}

Keywords Opportunity-use model of student learning · Network analysis · Interaction process $\cdot$ Classroom

\section{Einleitung}

Unterricht kann als ein komplexes soziales und dynamisches System gesehen werden, in welchem sich die beteiligten Personen (Schüler*innen und Lehrkräfte) gegenseitig beeinflussen (Vieluf et al. 2020). Ein theoretisches Rahmenmodell, das die Komplexität von Unterricht abbildet und gleichzeitig verschiedene Einflussfaktoren auf schulisches Lernen bündelt, ist das häufig rezipierte und weiterentwickelte Angebots-Nutzungs-Modell (Fend 2008; Helmke 2009; Lipowsky 2020; Seidel 2014; Vieluf et al. 2020). Das Modell lenkt den Fokus auf diejenigen im Unterricht stattfindenden Prozesse, die das Lernen unterstützen (Pauli und Reusser 2006). Die Unterrichtsmerkmale wirken darin nicht deterministisch, sondern bilden Gelegenheitsstrukturen (Lernangebote) ab, die von den Schüler*innen aktiv wahrgenommen und genutzt werden müssen, um eine entsprechende Wirkung zu erzielen (Lipow- 
sky 2020). Im aktuellen, integrativen Angebots-Nutzungs-Modell von Vieluf et al. (2020) manifestiert sich die Nutzung in Form von mentalen Prozessen, bei welchen sich Kognitionen sowie motivationales und emotionales Erleben ständig gegenseitig beeinflussen (Vieluf et al. 2020). Diese mentalen Prozesse spiegeln sich im Unterrichtshandeln wider (Seidel 2020; Vieluf et al. 2020), das wiederum andere am Unterricht beteiligte Personen beeinflusst. Das Verhalten der Schüler*innen stellt demnach einen Indikator für die Nutzung und gleichwohl auch ein potenzielles, situationales Lernangebot für die Mitschüler*innen dar. Das integrative AngebotsNutzungs-Modell hebt demnach den interaktiven Charakter des Unterrichts besonders hervor. Um die Nutzung von Lernangeboten durch Schüler*innen im Sinne dieses integrativen Modells in empirischen Studien zu erfassen, könnte die Analyse der ko-konstruktiven Interaktionsprozesse eine gewinnbringende Perspektive für die Unterrichtsforschung eröffnen, da die Beteiligung an der Interaktion die Nutzung der individuellen Schüler*innen auf Prozessebene sichtbar macht und dabei ebenso die potenziellen Lernangebote, die den Schüler*innen durch die Beteiligung der anderen ermöglicht werden, berücksichtigt werden. So könnte der Wechselwirkung von Angebot und Nutzung, die in dem Modell dargestellt wird, entsprechend begegnet werden.

Die Analyse ko-konstruktiver Interaktionsprozesse im Unterricht steht jedoch vor methodischen Herausforderungen, da die Akteur*innen in einer dynamischen Beziehung zueinander stehen und sich ständig gegenseitig beeinflussen. Es bedarf demnach Methoden, die die Wechselwirkung zwischen den beteiligten Personen im zeitlichen Verlauf adäquat abbilden. Im Rahmen dieses Artikels wird die Netzwerkanalyse als möglicher Ansatz vorgestellt und anhand erster Analysen das Potenzial dieser Methode für die Beschreibung und Analyse manifester Nutzungsprozesse aufgezeigt, die sich in der Ko-Konstruktion widerspiegeln.

\section{Ko-Konstruktionen im Unterricht}

Das Kernelement der Ko-Konstruktion ist die soziale Interaktion von Individuen. So wird aus einer sozial-konstruktivistischen Perspektive auf Lernen (Vygotsky 1978) diesen sozialen Interaktionen im Unterricht eine zentrale Bedeutung insbesondere beim Aufbau von Wissen beigemessen, da diese die individuelle Entwicklung und das Lernen von Individuen formen (Vygotsky 1978). Unter Interaktion wird dabei ,das wechselseitige Aufeinanderwirken im Wahrnehmen, Beurteilen, Kommunizieren und Beeinflussen“ (Hofer 1997, S. 213) von Personen im Unterricht verstanden. Individuen handeln ko-konstruktiv auf Basis ihres jeweiligen Vorwissens und ihrer unterschiedlichen Perspektiven auf den Lerngegenstand gemeinsam mit anderen Personen eine Lösungsstrategie für ein Problem aus (Pauli und Reusser 2000; Webb 2009). Nach Vygotsky (1978) bedeutet dies, dass das eigene Vorwissen der Lernenden innerhalb eines kommunikativen Prozesses durch das Wissen der anderen Gesprächsteilnehmenden erweitert und somit neues Wissen innerhalb von Interaktionsprozessen ko-konstruiert wird (Ackermann 2011). Das ko-konstruierte Wissen wird dann von den Lernenden internalisiert und somit zu einem Bestandteil der eigenen kognitiven Strukturen. In der Interaktion mit kompetenteren anderen 
(Lehrkräfte oder Mitschüler*innen) können die Schüler*innen individuell in ihrer Zone der nächsten Entwicklung (Vygotsky 1978) unterstützt werden, die als ,,[d]as Gebiet der noch nicht ausgereiften, jedoch reifenden Prozesse“ beschrieben wird (Vygotsky 1978, S. 83). Schüler*innen können die ihnen gestellten Aufgaben oder Problemstellungen diesem theoretischen Ansatz nach noch nicht selbstständig, aber mit Unterstützung eines bzw. einer fähigeren anderen bewältigen.

Für die Ko-Konstruktion von Wissen ist die aktive Partizipation der Schüler*innen von Bedeutung (Mercer und Dawes 2014; Vygotsky 1978). Um neues Wissen aufzubauen, sollten Schüler*innen sich daher aktiv an Gesprächen beteiligen und Diskussionspunkte und Lösungsvorschläge nicht einfach annehmen oder ignorieren, sondern diese aufgreifen, mit ihrem eigenen Vorwissen verknüpfen und weiter erörtern (Nemeth et al. 2019). So wird ihnen ermöglicht, Bedeutungen und neues Wissen in einem Interaktionsprozess inhaltsbezogen gemeinsam zu konstruieren.

Die vorangegangenen Ausführungen zum sozial-konstruktivistischen Lernverständnis machen deutlich, dass nicht nur Lehrkräfte, sondern auch Schüler*innen eine zentrale Rolle in der Gestaltung des Unterrichts bzw. des Lernprozesses einnehmen - Lehrkräfte und Schüler*innen sowie die Schüler*innen untereinander schaffen gemeinsam in der Interaktion eine ,Lernumgebung als Raum von Lerngelegenheiten“ (Klieme 2006, S. 765). Diese Lerngelegenheiten können im Sinne des integrierten Angebots-Nutzungs-Modells (Vieluf et al. 2020) ein Lernangebot darstellen, welches von den Schüler*innen wahrgenommen und genutzt werden kann. In der sozialen Interaktion findet demnach ein ständiger Wechsel aus Angebots- und Nutzungsprozessen statt (Ackermann 2011) - die Konstrukte stehen in einer dynamisch reziproken Beziehung zueinander. Inwiefern ein Zusammenhang zwischen der aktiven Beteiligung an der Ko-Konstruktion und den mentalen Prozessen (Nutzung von Lernangeboten) besteht, wird im Folgenden anhand von Studienergebnissen erläutert.

\subsection{Forschungsergebnisse zum Zusammenhang von aktiver Beteiligung an der Ko-Konstruktion und mentalen Prozessen bei Schüler*innen}

Studien, die die verbale Partizipation der Schüler*innen im Unterricht untersuchen, konnten zeigen, dass die aktive Teilnahme am Unterrichtsgespräch mit der kognitiven Involviertheit zusammenhängt (Jurik et al. 2013; Sedláček und Šed'ova 2020). Schüler*innen, die höhere kognitive Involviertheit im Unterricht berichten, sind auch insgesamt über die gesamte Unterrichtszeit hinweg länger am Klassengespräch beteiligt (Sedláček und Šed'ova 2020). Das Melden (als Anreiz, sich mündlich beteiligen zu wollen) während des Klassengesprächs steht ebenso in Zusammenhang mit der kognitiven Involviertheit der Lernenden (Böheim et al. 2020) und kann den Lernerfolg der Schüler*innen positiv beeinflussen (Decristan et al. 2019). Pauli und Lipowsky (2007) zeigen, dass sich eine stärkere Beteiligung am Klassengespräch (gemessen über die Anzahl an Redebeiträgen) positiv auf das kognitive und motivational-emotionale Selbsterleben der Lernenden auswirkt. Auch Hijzen et al. (2007) identifizieren die aktive Beteiligung an lerngegenstandsbezogenen Interaktionsprozessen als lernförderlich. Hierüber hinaus wirkt sich auch die Qualität der kokonstruktiven Interaktionsprozesse positiv auf die Leistungsentwicklung der Schü- 
ler*innen aus. So fanden Battistich et al. (1993) in einer Untersuchung von kooperativen Gruppenarbeitsphasen heraus, dass Schüler*innen von einem häufigen Einsatz dieser Methode bei entsprechender Interaktionsqualität (z. B. manifestiert durch gegenseitige Hilfestellungen, gemeinsame Aufgabenbearbeitung, gutes Gruppenklima) bzgl. ihrer Kompetenzentwicklung profitieren (Nemeth et al. 2019). Es kann also zusammenfassend festgehalten werden, dass die kognitive Involviertheit der Schüler*innen unter dieser Perspektive einerseits durch die soziale Interaktion ausgelöst wird und sich andererseits in der sozialen Interaktion mit anderen manifestiert.

\subsection{Erfassung der Beteiligung an ko-konstruktiven Interaktionsprozessen}

Um die Beteiligung an der sozialen Interaktion als Indikator für die Nutzung von Lernangeboten erfassen zu können, hat sich in der aktuellen empirischen Unterrichtsforschung die (Video-)Beobachtung als Datenerhebungsverfahren bewährt (z.B. Pauli und Reusser 2006). Über die hierdurch gewonnenen Beobachtungsdaten ist es möglich nachzuvollziehen, was sich im Unterricht auf welche Art und Weise ereignet und wie die Akteur*innen im Unterricht miteinander interagieren.

Die aktive Beteiligung an den ko-konstruktiven Interaktionsprozessen wird häufig anhand von Redebeiträgen, die die Personen einbringen, erfasst und über deren Häufigkeit quantifiziert (Ackermann 2011; Herrmann et al. 2021). Als Redebeitrag werden dabei zumeist einzelne Gesprächsbeiträge definiert, wobei diese durch einen Sprecher*innen- oder Adressat*innenwechsel begrenzt werden (Herrmann et al. 2021). Über eine Quantifizierung hinaus kann die Qualität der Redebeiträge beispielsweise durch das kognitive Anspruchsniveau und die Verwendung elaborierter Strategien bestimmt werden (Nemeth et al. 2019). Die Redebeiträge werden dabei oftmals statisch und ohne Adressant*innen erfasst (Perrez et al. 2006). Die gerichteten, reziproken und dynamischen Interaktionsprozesse, also die Ko-Konstruktion von Wissen in der sozialen Interaktion, werden demnach häufig nicht berücksichtigt. Einzig die Studie von Nemeth et al. (2019) ging der Frage nach, in welchem Maß die Lernenden während des kooperativen Lernens gegenseitig aufeinander Bezug nehmen und welche Indikatoren ko-konstruktiver Aktivitäten sich ableiten lassen. Die Autor*innen analysierten alle aufgabenbearbeitungsbezogenen Redebeiträge der Schüler*innen während einer kooperativen Lernphase dahingehend, ob sie einen Bezug zum Redebeitrag eines anderen Gruppenmitglieds aufweisen oder nicht (Nemeth et al. 2019). Gründe für die oben adressierte Forschungslücke könnten der hohe Anspruch an die kleinschrittigen, detaillierten Beobachtungsaufträge sowie die aufwändigen Analysemethoden sein, die nötig wären, um solch komplexe und dynamische Interaktionsprozesse zu erfassen.

\subsection{Anforderungen an die Erfassung ko-konstruktiver Interaktionsprozesse}

Um ko-konstruktive Interaktionsprozesse zu erfassen, ist es von Relevanz zu identifizieren, ob ein*e Schüler*in in seinem*ihrem Redebeitrag Bezug auf vorherige Redebeiträge nimmt und wie sich das Gespräch im Verlauf der Zeit bezüglich der Qualität entwickelt - bzw. inwiefern gemeinsam in der Interaktion neues Wissen ko-konstruiert wird. Es geht also unter anderem um die Analyse von Strukturen 
und Mustern des Interaktionsprozesses. Liegt das Forschungsinteresse zudem darin zu untersuchen, ob die Beteiligung an der ko-konstruktiven Wissensbildung einen positiven Einfluss auf die Leistungsentwicklung der Schüler*innen hat, ist es ebenso von Bedeutung zu berücksichtigen, wie häufig und in welcher Qualität die Schüler*innen ein potenzielles Lernangebot (hier: operationalisiert als Redebeitrag, den der*die Schüler*in empfangen hat) erhalten haben. Denn ko-konstruktive Lernprozesse werden als Wechselspiel aus Angebots-Nutzungs-Prozessen, die in einer dynamischen, reziproken Beziehung zueinanderstehen, verstanden. Es ist also zudem relevant zu erfassen, welche*r der Schüler*innen überhaupt ein Lernangebot erhalten hat (differenzielles Lernangebot), das genutzt und auf welches dann wiederum Bezug genommen werden kann. Je nachdem, wie häufig ein*e Schüler*in demnach von der Lehrkraft oder den Mitschüler*innen mit einem (elaborierten) Redebeitrag adressiert wird, erhalten die Lernenden ein individuelles (bzw. differenzielles) Lernangebot (Decristan et al. 2019). Dies kann sich im klassenöffentlichen Unterrichtsgespräch beispielsweise durch Aufrufe der Lehrkraft nach einer Frage (Decristan et al. 2019) oder in Gruppenarbeitsphasen durch die (direkte) Ansprache eines oder mehrerer Gruppenmitglieder zeigen.

Um diesen Anforderungen gerecht zu werden, benötigt es idealtypisch einen methodischen Zugang, der die Interaktion zwischen den Akteur*innen im Verlauf der Unterrichtszeit adäquat erfassen und zusätzlich die individuelle Stellung der Person im ko-konstruktiven Interaktionsprozess berücksichtigen kann. Die netzwerkbasierte Analyse von Videobeobachtungen könnte sich eignen, um diesen Anforderungen zu begegnen. In diesem methodischen Ansatz werden die in den Videodaten beobachteten Interaktionen im zeitlichen Verlauf des Unterrichts in einem (dynamischen) Netzwerk subsumiert und anschließend statistisch analysiert.

\section{Die videobasierte Netzwerkanalyse als Forschungsansatz zur Analyse von ko-konstruktiven Interaktionsprozessen}

Um die (differenzielle) Beteiligung an ko-konstruktiven Interaktionsprozessen im Detail verstehen und analysieren zu können, ist es folglich nicht nur von Bedeutung zu erfassen, wer an der Bearbeitung einer Lernaufgabe beteiligt ist, wer die aktiven und wer die peripheren Akteur*innen sind (de Laat et al. 2007), sondern auch, wer mit wem gemeinsam an einer Lösungsfindung beteiligt ist (Interaktion zwischen Akteur*innen), wer konkret ein Lernangebot erhält und wie sich die Beteiligung (im Sinne von Mustern) über die Zeit verändert (dynamische Entwicklung der Interaktionsprozesse). Andere Methoden, wie beispielsweise die reine Auszählung von Redebeiträgen über Beobachtungen, bei denen die Adressat*innen vernachlässigt werden, oder auch die subjektive Einschätzung der eigenen Beteiligung über Fragebögen, ermöglichen es nicht, die Gestaltung der ko-konstruktiven Prozesse bzw. die Muster der Prozesse zwischen den Akteur*innen systematisch und im Detail zu analysieren (de Laat et al. 2007). Sie fokussieren eher Eigenschaften und Zuschreibungen von Individuen, die in einem sozialen Gefüge agieren.

Die Netzwerkanalyse hingegen fokussiert nicht die Individuen an sich, sondern die Beziehung zwischen den Personen - und nutzt diese als Datenquelle (de Laat 
et al. 2007; Mejeh und Hascher 2021; Wagner und González-Howard 2018). Sie bildet das soziale Umfeld (hier: den Unterricht mit seinen Akteur*innen) demnach als Muster von Beziehungen (hier: Interaktionen bzw. gerichtete Redebeiträge) zwischen den interagierenden Akteur*innen ab (Wasserman und Faust 1997). Die Netzwerkanalyse geht also über die reine Häufigkeitsbestimmung von Redebeiträgen hinaus und ermöglicht es, die reziproke Beziehung (Ko-Konstruktionsprozesse) zwischen zwei oder mehr Akteur*innen im Unterricht zu analysieren (Bokhove 2018; Butts 2008; Grunspan et al. 2014; Li et al. 2011). Für erste empirische Studien im Bereich der Lehr-Lern-Forschung, die auf diesen Ansatz zurückgreifen, sei auf Bokhove (2018), Ryu und Lombardi (2015) und de Laat et al. (2007) aus Platzgründen lediglich verwiesen. Im Folgenden wird die Netzwerkanalyse mit ihren Ansätzen und Kennwerten für die Analyse von Interaktionsprozessen im Unterricht knapp vorgestellt ${ }^{1}$.

Ein Netzwerk besteht aus Knoten (hier: Schüler*innen und Lehrkraft) und Kanten (hier: die gerichteten Redebeiträge zwischen den Akteur*innen). Bei der Analyse von Interaktionsprozessen werden die Kanten als zeitlich charakterisiert; das heißt, neben der gerichteten Information, wer (Sender*in) wem (Empfänger*in) etwas kommuniziert, wird ebenso der Beginn und das Ende der Interaktion in den Netzwerkdaten erfasst. Das daraus resultierende dynamische Netzwerk kann als Ereignisnetzwerk betrachtet werden, das durch sich schnell verändernde Ereignisse zwischen den einzelnen Akteur*innen des Netzwerks gekennzeichnet ist. Ereignisnetzwerke sehen je nach Messzeitpunkt folglich sehr unterschiedlich aus. Daten eines solchen Netzwerks, welche isoliert zu einem spezifischen Zeitpunkt des Zeitverlaufes betrachtet werden, haben sehr wenig Aussagekraft, wenn die Interaktionen zwischen den beteiligten Akteur*innen in ihrer Gesamtheit beschrieben und analysiert werden sollen (Steglich und Knecht 2010). Eine analytische Annäherung an dynamische Netzwerke könnte daher zeitaggregiert (statisch) erfolgen, indem alle Ereignisse, die in einem bestimmten Zeitraum stattfinden, in einem gewichteten und gerichteten Netzwerk zusammengefasst werden (Sulo et al. 2010) oder indem eine explizite (längsschnittliche) Adressierung zeitlicher Veränderungen der Ereignisse in den Fokus gerückt wird (Steglich und Knecht 2010).

Ein Kennwert der Netzwerkanalyse, welcher auch für die Analyse der ko-konstruktiven Interaktionsprozesse im Unterricht von Bedeutung ist, ist die Zentralität (Fuhse 2018). Die Zentralität gibt Auskunft über das Verhalten der einzelnen Akteur*innen innerhalb eines Netzwerks. Sie zeigt das Ausmaß an, in dem ein Individuum mit anderen Akteur*innen des Netzwerks interagiert (Wasserman und Faust 1997). Dabei wird in der einfachsten Annäherung die Anzahl der Verbindungen mit den anderen Mitgliedern gemessen (Degree, Knotengrad) und in den „Indegree“ (hier: Anzahl der empfangenen Redebeiträge) und den „Outdegree“ (hier: Anzahl der gesendeten Redebeiträge) $)^{2}$ überführt. Im Rahmen der Netzwerkanalyse können Personen als zentral angesehen werden, wenn sie besonders häufig oder auch besonders

\footnotetext{
1 Eine ausführliche Beschreibung des methodischen Ansatzes findet sich bei de Laat et al. (2007), Bokhove (2018), Wagner und González-Howard (2018) und Mejeh und Hascher (2021).

${ }^{2}$ Eine ausführlichere Erläuterung der Kennwerte findet sich im Methodenteil des Artikels.
} 
Abb. 1 Beispielhafte Darstellung eines gewichteten Netzwerks, bestehend aus vier Schüler*innen (bezeichnet mit $S 1$ bis S4)

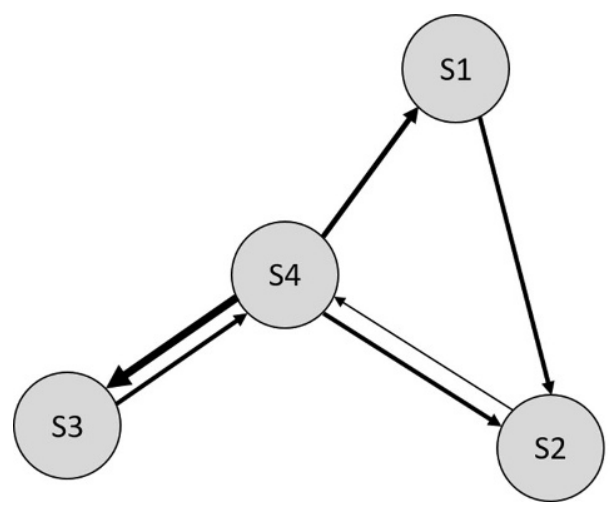

lange mit anderen Akteur*innen des Netzwerks interagieren - periphere Personen im Netzwerk hingegen beteiligen sich eher wenig an der Interaktion (Fuhse 2018).

Ein weiteres wesentliches Merkmal von Netzwerkanalysen ist die Möglichkeit der Visualisierung von Beziehungen innerhalb des Netzwerks in Soziogrammen (Abb. 1). Dabei können sowohl Knoten als auch Kanten gewichtet werden, indem die Größe der Radien oder auch die Dicke der Kanten variiert wird. Visualisierungen von sozialen Netzwerken zeigen, ob Interaktionen zwischen allen Akteur*innen eines Netzwerks stattfinden und ob einige Akteur*innen mehr (oder weniger) mit anderen Individuen kommunizieren (Haythornthwaite 2001). Auf diese Weise kann die Struktur der Interaktionen innerhalb des Netzwerks als auch die Position einzelner Personen - spielen sie eine zentrale oder periphere Rolle im Netzwerk untersucht werden.

Zusammenfassend kann festgehalten werden, dass sich die Netzwerkanalyse als eine ergänzende Möglichkeit zur Darstellung von Interaktionsprozessen in der empirischen Unterrichtsforschung eignen könnte, da sie eine relationale Art der Betrachtung der Aktivitäten der Akteur*innen im Unterricht bietet und hierdurch teils erheblich über etablierte Verfahren, wie beispielsweise retrospektive Fragebogenerhebungen, hinausgeht (Bokhove 2018; Butts 2008; Froehlich et al. 2020; Grunspan et al. 2014; de Laat et al. 2007; Li et al. 2011).

\section{Die vorliegende Studie}

Soziale Interaktionen im Unterricht formen das Lernen der Schüler*innen. In ihnen manifestieren sich die kognitiven Nutzungsprozesse der Schüler*innen. Außerdem ermöglichen sie fortlaufend Lernangebote, die die Schüler*innen wahrnehmen und nutzen können und auf welche Mitschüler*innen entsprechend reagieren können. Angebot und Nutzung stehen demnach in einer dynamischen Wechselbeziehung zueinander. Um die Nutzung zu erfassen, könnte die Analyse der ko-konstruktiven Interaktionsprozesse ein gewinnbringender Ansatz sein, da genau diese Wechselwirkung in den gerichteten Redebeiträgen der Schüler*innen sichtbar wird: sie bilden die manifesten Nutzungsprozesse sowie potenzielle Lernangebote für Mitschü- 
ler*innen ab. Im Rahmen dieses Artikels wird am Beispiel einer Gruppenarbeitsphase der Ansatz der Netzwerkanalyse mit videografierten Unterrichtsdaten als ein methodischer Zugang zur Modellierung von ko-konstruktiven Interaktionsprozessen vorgestellt. Anhand erster Analysen wird das Potenzial der Methode aufgezeigt und ein Ausblick auf einen potenziellen, zukünftigen Mehrwert verdeutlicht. Folgende übergreifende, eher methodische Fragestellung ist für diesen Beitrag handlungsleitend: Inwiefern können durch Netzwerkanalysen konstituierende Merkmale ko-konstruktiver Interaktionsprozesse im Unterricht beschrieben und analysiert werden?

Um sich dieser Fragestellung anzunähern, werden folgende sechs inhaltliche Fragestellungen adressiert:

1. In welchem Umfang beteiligen sich die einzelnen Schüler*innen aktiv am Interaktionsprozess (als Indikator der Nutzung sowie eines potenziellen Lernangebots für Mitschüler*innen)?

2. In welchem Umfang erhalten die Schüler*innen ein Lernangebot (potenziell empfangene Redebeiträge)?

3. Gibt es Schüler*innen, die eher eine zentrale oder periphere Rolle einnehmen, oder sind die Netzwerke bezüglich der Beteiligung der Schüler*innen homogen?

4. Bei Heterogenität: Welche Schüler*innen (bezogen auf deren Hintergrundmerkmale, wie z.B. die Fachnote) nehmen eine zentrale oder periphere Rolle ein?

5. Inwiefern nehmen die Schüler*innen in ihrer Interaktion Bezug aufeinander?

6. Wie entwickelt sich der ko-konstruktive Interaktionsprozess über die gesamte Gruppenarbeitsphase?

\section{Methode}

\subsection{Datengrundlage}

Um den methodischen Ansatz der Netzwerkanalyse für die Analyse von ko-konstruktiven Interaktionsprozessen im Unterricht vorzustellen, wird eine videografierte Deutschstunde einer dritten Grundschulklasse herangezogen. Der Unterricht in dieser Klasse wurde mittels eines schüler*innenzentrierten Aufnahmesystems videografiert (Troll et al. 2020). Dabei werden ergänzend zu der Lehrkraftkamera und der Überblickskamera an jedem Gruppentisch weitere Kameras aufgestellt, um die Handlungen und Interaktionen der Schüler*innen im Detail erfassen zu können.

Die Deutschstunde dauert 39 min und kann dem Kompetenzbereich „Sprechen und Zuhören" der Bildungsstandards für das Fach Deutsch zugeordnet werden: $\mathrm{Zu}$ Beginn liest die Lehrkraft den Schüler*innen eine Geschichte über ein Fabelwesen vor (7 min). Sie sollen aufmerksam zuhören, um jenes im Anschluss in Partnerarbeit aus dem Gedächtnis heraus zeichnen zu können (9 min). Es folgt eine Gruppenarbeitsphase, in der je zwei Partnerteams gemeinsam einen Steckbrief über das Fabelwesen ausfüllen (12 min). Dabei sollen die Schüler*innen im Viererteam Besonderheiten des Wesens begründet herausarbeiten. Im Anschluss werden im Plenum Zuhör- und Merkstrategien diskutiert (5 min). Insgesamt werden 6 min für Übergänge und Sozialformwechsel benötigt. 
Zur Veranschaulichung des methodischen Ansatzes wurde die zwölfminütige Gruppenarbeitsphase ausgewählt, in welcher die Lernenden in sechs Gruppen á vier Schüler*innen gemeinsam den Arbeitsauftrag erfüllen. Die Lehrkraft begleitet den Gruppenprozess in der Zeit und ist daher immer wieder in die Interaktionsprozesse der Schüler*innengruppen involviert. Größtenteils geht sie von Gruppentisch zu Gruppentisch und gibt den Schüler*innen kleine Hilfestellungen, um den Lösungsprozess zu unterstützen. Diese Phase wird den Netzwerkanalysen zugrunde gelegt, da in kollaborativen Arbeitsphasen, wie es hier der Fall ist, eine ko-konstruktive Wissensbildung durch ,den Austausch verschiedener Herangehensweisen, die Möglichkeit Fragen zu stellen und Erklärungen zu erhalten“ erreicht werden kann (Nemeth et al. 2019, S. 53).

\subsection{Stichprobe}

Die Personenstichprobe umfasst insgesamt 24 Schüler*innen, wobei aufgrund technischer Probleme (z. B. Ausfall der Videokamera) lediglich 20 Schüler*innen in fünf Gruppen in die Analysen eingeschlossen werden. Da die Schüler*innen zum Zeitpunkt der Aufnahmen noch keine Ziffernnoten erhielten, schätzt die Lehrkraft die Schüler*innen vor der Aufnahme hinsichtlich ihrer Leistung ein und vergibt jedem Kind eine Fachnote. Acht der Schüler*innen erhalten von der Lehrkraft im Fach Deutsch eine 1 oder 2 (leistungsstärker), zwölf der Schüler*innen die Note 3 oder 4 (leistungsschwächer).

\subsection{Instrument}

Für eine netzwerkbasierte Analyse der ko-konstruktiven Interaktionsprozesse ist es relevant, die Beziehung (hier: die gerichteten Redebeiträge) der Akteur*innen im Unterricht zu erfassen. Um diese interpretieren zu können, ist es notwendig, den Gegenstand der Interaktionen zu berücksichtigen. Die vorliegenden schüler*innenzentrierten Unterrichtsvideos der Deutschstunde wurden daher mittels eines niedrig bis mittel-inferenten Kategoriensystems im Event-Sampling-Verfahren kodiert $^{3}$. In Anlehnung an Nemeth et al. (2019) wurde ein Kategoriensystem entwickelt (Abb. 2), das alle Redebeiträge nach ihrer Art bzw. ihrem Zweck klassifiziert, um die Beziehungen (also die gerichteten Redebeiträge) inhaltlich bestimmen zu können. Die Redebeiträge der Schüler*innen und die der Lehrkraft wurden mit dem gleichen Kategoriensystem eingeschätzt. Ein neuer Redebeitrag wurde immer nach einem Sprecher*innenwechsel oder wenn der*dieselbe Schüler*in bzw. die Lehrkraft eine mindestens drei Sekunden andauernde Pause machte bzw. ein neues

\footnotetext{
${ }^{3}$ Im zusätzlichen Onlinematerial wird ein Transkriptausschnitt bereitgestellt, welcher eine beispielhafte Kodierung eines Gesprächs zwischen den Schüler*innen und der Lehrkraft veranschaulicht (Onlinematerial 1).
} 


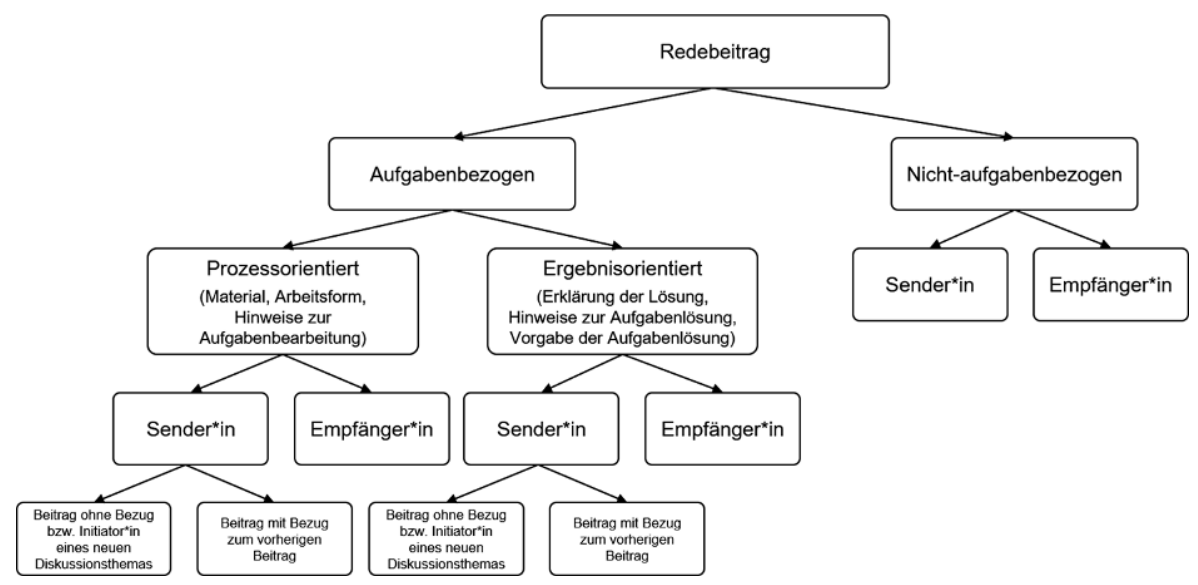

Abb. 2 Übersicht über das Kategoriensystem in Anlehnung an Nemeth et al. (2019)

Thema ansprach, kodiert (Nemeth et al. 2019; Pauli 2006). Das Kategoriensystem ${ }^{4}$ unterscheidet zwischen nicht-aufgabenbezogenen Redebeiträgen (z. B. „Ist in deiner Trinkflasche Apfelsaftschorle?") und aufgabenbezogenen Redebeiträgen. Die aufgabenbezogenen Redebeiträge wurden in zwei Ebenen der Auseinandersetzung unterteilt: Prozessorientiert (z. B. „Ich möchte aufschreiben, du kannst mir diktieren, was ich schreiben soll.“) und Ergebnisorientiert (z.B. „Das Fabelwesen, das wir beschreiben sollen, hat abstehende Ohren und zwei lange Beine, die es ausfahren kann. Das ist eine Besonderheit, wir Menschen haben das nicht"). Ein potenzielles Lernangebot für die Schüler*innen äußert sich dabei am ehesten in einem aufgabenbezogenen, ergebnisorientierten Redebeitrag der Schüler*innen, da diese Kategorie auch elaborierte und kognitiv anregende Beiträge, die zur Lösung der Aufgabe führen, einschließt. Jedoch werden die Beiträge der Schüler*innen nicht nach ihrer Qualität beurteilt, weshalb diesbezüglich keine endgültige Aussage getroffen werden kann. Für eine erste Annäherung werden die aufgabenbezogenen, ergebnisorientierten Redebeiträge jedoch trotzdem als ein potenzielles Lernangebot interpretiert.

Um die Beziehung der Akteur*innen abzubilden, wurde zudem kodiert, wer (Sender*in) wen (Empfänger*in) mit einem Redebeitrag adressierte. Adressierte die Lehrkraft beispielsweise die gesamte Klasse, da sie einen Arbeitsauftrag erteilte oder einen Hinweis bezüglich der Bearbeitung des Arbeitsblattes gab, wurde als Empfänger*in des Redebeitrags jede*r Schüler*in kodiert. Dasselbe gilt, wenn innerhalb einer Gruppe ein*e Schüler*in einen Redebeitrag an alle Gruppenmitglieder richtet, indem sie*er beispielsweise sagt, „, Guckt mal alle, beide Partnerteams ha-

\footnotetext{
${ }^{4}$ Es handelt sich dabei nicht um Kategorien, die die Qualität der Redebeiträge einschätzen; vielmehr werden diese hier lediglich nach ihrer Art bzw. nach ihrem Zweck klassifiziert. Um die Qualität der Redebeiträge - wie z. B. das kognitive Anspruchsniveau oder die Verwendung elaborierter Strategien, Nemeth et al. (2019) - zu erfassen, müssten die Redebeiträge dahingehend re-analysiert werden. Für die Kodierung der Qualität der Redebeiträge könnte beispielsweise auf das Kategoriensystem SEDA von Hennessy et al. (2016) verwiesen werden.
} 
ben das Fabelwesen ganz unterschiedlich gezeichnet. Das ist lustig “. Spricht ein*e Schüler*in nur einen Teil der Gruppe an, wie in folgendem Beispiel, „Elias, Mia, was denkt ihr ist denn eine Besonderheit von dem Fabelwesen? Wir glauben ja, dass es die spitzen Ohren sind. ", dann werden auch nur die zwei Schüler*innen als Empfänger*innen des Redebeitrags kodiert. Murmelt ein Kind etwas vor sich hin oder wiederholt einen Beitrag ohne direkt eine andere Person zu adressieren, wird als Empfänger*in des Beitrags das Kind selbst gewählt. Sind die Empfänger*innen eines Redebeitrags nicht ersichtlich, wird ein „missing value“ vergeben. In Anlehnung an Nemeth et al. (2019) wurden die gesendeten Redebeiträge zudem dahingehend klassifiziert, ob sie einen Bezug zu einem vorherigen Redebeitrag aufweisen (z.B. „Stimmt, da hast du Recht, ich habe auch gehört, dass das Fabelwesen nur drei Finger hatte. ") oder ob es sich dabei um einen Redebeitrag ohne Bezug (z. B. „So, jetzt müssen wir noch die Besonderheiten des Fabelwesens eintragen. ") handelt.

\subsection{Kodierer*innenschulung und -übereinstimmung}

Die Kodierung der Redebeiträge wurde von drei geschulten Kodierer*innen sowie der Erstautorin des Beitrags vorgenommen. Die zehnstündige Kodierer*innenschulung erstreckte sich über zwei Tage. In der Schulung lernten die Kodierer*innen zunächst das Aufnahmesystem kennen, erhielten Input zum Kategoriensystem sowie der dahinterliegenden Theorie. Nach der Erläuterung des methodischen Vorgehens folgte eine erste gemeinsame Probekodierung mit anschließenden Übungsphasen und intensiven Diskussionsrunden. Aufgrund einer geringen Beurteiler*innenübereinstimmung (Cohen's Kappa <0,50) der unabhängig voneinander kodierten Sequenzen erfolgte am zweiten Schulungstag eine Diskussion des Kategoriensystems auf Basis identifizierter Ankerbeispiele der Beurteiler*innen (kriteriale Validität). Nach erneuter Übung und umfangreicher Diskussion der Ergebnisse (diskursive Validität) wurde das Training mit guten (Art des Redebeitrags) bis sehr guten (Empfänger*innen des Redebeitrags) Übereinstimmungswerten (Tab. 1) abgeschlossen (Döring und Bortz 2016, S. 346). Die gesamte Kodierung der Redebeiträge der individuellen Schüler*innen und der Lehrkraft wurde über die gesamte Unterrichtszeit hinweg einzeln von je einem*einer Kodierer*in vorgenommen.

Tab. 1 Übereinstimmungen zwischen Masterkodierung und trainierten Beurteiler*innen bei der Kodierung des Interaktionsverhaltens

\begin{tabular}{|c|c|c|c|c|}
\hline & \multicolumn{2}{|c|}{ Redebeitrag } & \multicolumn{2}{|c|}{ Empfänger*in des Redebeitrags } \\
\hline & $\begin{array}{l}\text { Cohen's } \\
\text { Kappa }\end{array}$ & $\begin{array}{l}\text { Prozentuale Überein- } \\
\text { stimmung }(\%)\end{array}$ & $\begin{array}{l}\text { Cohen's } \\
\text { Kappa }\end{array}$ & $\begin{array}{l}\text { Prozentuale Überein- } \\
\text { stimmung }(\%)\end{array}$ \\
\hline Kodierer*in 1 & 0,65 & 84,97 & 0,83 & 90,90 \\
\hline Kodierer*in 2 & 0,69 & 86,82 & 0,81 & 92,06 \\
\hline Kodierer*in 3 & 0,63 & 84,46 & 0,83 & 92,74 \\
\hline
\end{tabular}

$592 \mathrm{~s}$ wurden von allen Kodierer*innen unabhängig voneinander kodiert, um die Übereinstimmung zu berechnen; Fleiss KappaRedebeitrag $k=0,64$; Fleiss KappaEmpfänger*in $k=0,80$ 


\subsection{Datenanalyse}

Die kodierten Gesprächsdaten wurden in $\mathrm{R}$ unter Nutzung der Pakete ndtv (Version 0.13.0) und statnet (Version 4.3.0) analysiert (Bender-deMoll und Morris 2019; Handcock et al. 2008). Für die Knoten der Schüler*innen wurden im Netzwerk die personenspezifischen, statischen Variablen ,,von der Lehrkraft eingeschätzte Leistung" sowie die Schüler*innen-ID hinterlegt. Für jede dynamische Kante (gerichteter Redebeitrag) wurde neben Start- und Endzeitpunkt der Interaktion hinterlegt, um welche Ebene der Auseinandersetzung (Prozessorientiert; Ergebnisorientiert; Nichtaufgabenbezogen) es sich handelt.

Fragestellung 1 In einem ersten Schritt werden die Häufigkeiten aller dyadischen Ereignisse (Anzahl der gerichteten Redebeiträge zwischen zwei Akteur*innen) bestimmt. Zur Berechnung der Zentralitätsmaße (In- und Outdegree) werden die kodierten Redebeiträge pro Gruppe in eine Häufigkeitsmatrix überführt. Wie intensiv sich eine Person an der Interaktion beteiligt (wie sehr sie ggf. das Lernangebot nutzt), kann mittels des Ausgangsgrads (Outdegree) berechnet werden. Dieser betrachtet die Häufigkeit der gesendeten Redebeiträge zu anderen Schüler*innen und der Lehrkraft, unabhängig von deren Dauer, über die gewählte Zeitspanne hinweg normiert an der Gesamtanzahl aller Redebeiträge in der Gruppe.

Fragestellung 2 Um ein Maß für die empfangenen Redebeiträge (potenzielles Lernangebot) zu generieren, wird der Eingangsgrad (Indegree) berechnet. Er beschreibt die Häufigkeit der empfangenen Redebeiträge über die gewählte Zeitspanne hinweg und ergibt sich aus der Summe aller empfangenen Redebeiträge ebenso normiert an der Gesamtanzahl aller Redebeiträge in der Gruppe. Dabei kann, wie bereits im Abschn. 5.3 Instrumente dargestellt, am ehesten ein aufgabenbezogener, ergebnisorientierter Redebeitrag als ein potenzielles Lernangebot interpretiert werden.

Fragestellung 3 Um die Rollen der Akteur*innen im Netzwerk interpretieren zu können, werden die In- und Outdegree-Werte näher betrachtet. Ein hoher OutdegreeWert ist ein Indikator für eine agile Person im Netzwerk, die häufig einen Redebeitrag sendet. Ein niedriger Outdegree-Wert hingegen ist ein Indikator für eine eher periphere Person im Netzwerk, die eher selten einen Redebeitrag einbringt. Um die Stellung der Akteur*innen im Netzwerk anschaulich zu verdeutlichen, wurden die In- und Outdegree-Werte in ein Soziogramm überführt. Die Dicke der gerichteten Kanten entspricht dabei der Anzahl der dyadischen Interaktionen zwischen den einzelnen Akteur*innen. Die Knoten der Graphen repräsentieren die einzelnen Schüler*innen und die Lehrkraft. Der Durchmesser der Knoten entspricht dem Totaldegree (Indegree und Outdegree zusammengenommen).

Fragestellung 4 Um die Rollen der Schüler*innen inhaltlich interpretieren zu können, werden den Knoten konkrete personenspezifische Eigenschaften zugeordnet. Die In- bzw. Outdegree-Werte des*der jeweiligen Schülers*in wurden daher mit der 
von der Lehrkraft eingeschätzten Fachnote korreliert ${ }^{5}$. Dies stellt jedoch nur eine erste Annäherung an eine systematische Interpretation der Struktur des Netzwerks dar.

Fragestellung 5 Um zu identifizieren, wie groß der Anteil an ko-konstruktiven Aktivitäten innerhalb der Interaktion ist, kann in Anlehnung an Nemeth et al. (2019) ein Bezugsindex sowohl auf Ebene der individuellen Schüler*innen als auch aggregiert auf Gruppenebene berechnet werden. Dieser ergibt sich aus dem prozentualen Anteil der Redebeiträge, die Bezug zu einem vorherigen aufweisen, gemessen an den gesamten Redebeiträgen. Dieser Index wird für jede der drei Ebenen der Auseinandersetzung mit dem Lerngegenstand berechnet.

Fragestellung 6 Um die Entwicklung der dynamischen Interaktionsprozesse zu beschreiben, werden erste zeitabhängige Visualisierungen und augenscheinliche Betrachtungen der Interaktionen der einzelnen Gruppen durchgeführt. Über die grafische Darstellung der Interaktionsprozesse im zeitlichen Verlauf ist es möglich, die Aktivität des Netzwerks unter Berücksichtigung der Ebenen der Auseinandersetzung über die gesamte Unterrichtszeit hinweg zu visualisieren und unterschiedliche Phasen im Interaktionsprozess herauszuarbeiten. Hierdurch können Entwicklungen bezüglich der aktiven Beteiligung der individuellen Schüler*innen und der gesamten Gruppe identifiziert werden.

\section{Ergebnisse}

Fragestellung 1 In Tab. 2 sind die deskriptiven Statistiken der In- und Outdegree-Werte dargestellt. Die Ausprägungen des Outdegree-Wertes zeigen, dass sich einige Schüler*innen im Mittel häufiger mit aufgabenbezogenen Redebeiträgen an der Gruppendiskussion beteiligen als ihre Mitschüler*innen (d.h. sie senden häufiger einen aufgabenbezogenen Redebeitrag). Einige Schüler*innen nutzen im Mittel das ihnen bereitgestellte Lernangebot demnach intensiver, wodurch diese für die anderen Gruppenmitglieder wiederum häufiger potenzielle Lernangebote ermöglichen. Die Spannbreite der aufgabenbezogenen Outdegree-Werte liegt zwischen $\operatorname{Min}_{\text {Ergebnisorientiert }}=0,05$ bis $\operatorname{Max}_{\text {Ergebnisorientiert }}=0,44$ sowie zwischen $\operatorname{Min}_{\text {Prozessorientiert }}=0,05$ bis $\operatorname{Max}_{\text {Prozessorientiert }}=0,41$.

Fragestellung 2 Wird der Indegree-Wert der aufgabenbezogenen, ergebnisorientierten Redebeiträge in Tab. 2 betrachtet, dann zeigt sich, dass im Mittel nicht alle Schüler*innen gleichermaßen ein potenzielles Lernangebot erhalten. Einige Schüler*innen werden häufiger durch einen aufgabenbezogenen, ergebnisorientierten Re-

\footnotetext{
5 Den Autor*innen liegen keine weiteren Hintergrundmerkmale der Schüler*innen vor. Aufgrund des exemplarischen Charakters der Studie ist die Fachnote als ein Hintergrundmerkmal der Schüler*innen vorerst ausreichend. In folgenden netzwerkanalytischen Studien wird die Einbeziehung weiterer Hintergrundmerkmale der Schüler*innen empfohlen.
} 
Tab. 2 Deskriptive Statistik der In- und Outdegree Werte für alle drei Ebenen der Auseinandersetzung

\begin{tabular}{llllll}
\hline Redebeitrag & & Min & Max & $M$ & $S D$ \\
\hline Ergebnisorientiert & Outdegree & 0,05 & 0,44 & 0,21 & 0,11 \\
& Indegree & 0,03 & 0,47 & 0,22 & 0,13 \\
Prozessorientiert & Outdegree & 0,05 & 0,41 & 0,22 & 0,11 \\
& Indegree & 0,05 & 0,45 & 0,23 & 0,12 \\
Nicht- & Outdegree & 0,07 & 0,52 & 0,24 & 0,13 \\
aufgabenbezogen & Indegree & 0,08 & 0,44 & 0,26 & 0,11 \\
\hline
\end{tabular}

$N=20$ Schüler*innen; Die Lehrkraft wurde von diesen Berechnungen ausgeschlossen

debeitrag adressiert als ihre Gruppenmitglieder, was ebenso in der Spannbreite des Indegree-Wertes deutlich wird: Min $_{\text {Ergebnisorientiert }}=0,03$ bis Max $_{\text {Ergebnisorientiert }}=0,47$.

Fragestellung 3 Um auf Ebene der Schüler*innen zu bestimmen, wer die zentralen und peripheren Akteur*innen im Netzwerk sind, können die individuellen Zentralitätsmaße der Schüler*innen betrachtet werden. Tab. 3 zeigt die Verteilung der Knotengrade für die gesendeten (Outdegree) und empfangenen (Indegree) Redebeiträge auf Schüler*innen- sowie der Lehrkraftebene in den jeweiligen Gruppen. Zudem zeigt diese, wie viele dyadische Ereignisse (Interaktion $_{\text {Gesamt }}=$ Anzahl der gerichteten Redebeiträge zwischen zwei Personen) überhaupt innerhalb der Gruppen kodiert wurden.

Beispielsweise beteiligen sich die Schüler*innen S8, S16, S7, S18 häufiger mit aufgabenbezogenen Redebeiträgen an der Gruppendiskussion als ihre Gruppenmitglieder. Diese Schüler*innen nutzen die ihnen gebotenen potenziellen Lernangebote in diesem Fall intensiver. Dadurch ermöglichen sie für ihre Gruppenmitglieder gleichzeitig auch häufiger ein potenzielles Lernangebot. Wird der Indegree-Wert auf Ebene der Schüler*innen betrachtet, so zeigt sich, dass Schüler*innen S2, S3, S4, S5, S8 und S18 häufiger Adressat*in eines aufgabenbezogenen, ergebnisorientierten Redebeitrags sind. Grundsätzlich zeigt sich jedoch, dass ein höherer Indegree mit einem höheren Outdegree der Schüler*innen einhergeht. Die signifikanten Korrelationen in Tab. 4 bestätigten dies auf allen Ebenen der Auseinandersetzung $\left(r_{\text {Ergebnisorientiert }}=0,52, p=0,020 ; r_{\text {Prozessorientiert }}=0,65, p=0,002 ; r_{\text {Nicht-aufgabenbezogen }}=0,58\right.$, $p=0,007)$. Schüler*innen, die häufiger direkt angesprochen werden, senden demnach auch häufiger einen Redebeitrag an ihre Gruppenmitglieder.

Abb. 3 veranschaulicht die Stellung der Personen im Netzwerk nochmals in einem Soziogramm. Die Dicke einer gerichteten Kante entspricht der Anzahl an gesendeten Redebeiträgen von dem*der entsprechenden Schüler*in bzw. der Lehrkraft, von dem*der der Pfeil (Kante) ausgeht. Alle gesendeten Redebeiträge einer Person zusammengenommen ergeben den Outdegree-Wert. Die Dicke der aufgabenbezogenen, ergebnisorientierten Kanten zeigt also, wie oft ein*e Schüler*in an eine bestimmte Person in der Gruppenarbeit einen Redebeitrag sendet bzw. - die Perspektive wechselnd - wie häufig ein*e Schüler*in von Mitschüler*innen oder der Lehrkraft einen Redebeitrag empfängt bzw. ein potenzielles Lernangebot erhält. Der Durchmesser der Knoten (der Schüler*innen oder der Lehrkraft; dieser bildet den TotaldegreeWert ab) subsumiert die Anzahl der gesendeten und empfangenen Redebeiträge. Je 
Tab. 3 Deskriptive Ergebnisse der netzwerkanalytischen Maße Indegree und Outdegree der Schüler*innen innerhalb der Gruppen

\begin{tabular}{|c|c|c|c|c|c|c|c|c|c|c|c|}
\hline \multirow{3}{*}{$\begin{array}{l}\text { Grup- } \\
\text { pe }\end{array}$} & \multirow{3}{*}{$\begin{array}{l}\text { Schüler } \\
\text { *in }^{\text {in }}\end{array}$} & \multirow{3}{*}{$\begin{array}{l}\text { Leis- } \\
\text { tungs- } \\
\text { gruppe }^{a}\end{array}$} & \multicolumn{3}{|c|}{ Ergebnisorientiert } & \multicolumn{3}{|c|}{ Prozessorientiert } & \multicolumn{3}{|c|}{ Nicht-aufgabenbezogen } \\
\hline & & & $\begin{array}{l}\text { Inter- } \\
\text { aktion }\end{array}$ & $\begin{array}{l}\text { Out- } \\
\text { degree }\end{array}$ & $\begin{array}{l}\text { In- } \\
\text { degree }\end{array}$ & $\begin{array}{l}\text { Inter- } \\
\text { aktion }\end{array}$ & $\begin{array}{l}\text { Out- } \\
\text { degree }\end{array}$ & $\begin{array}{l}\text { In- } \\
\text { degree }\end{array}$ & $\begin{array}{l}\text { Inter- } \\
\text { aktion }\end{array}$ & $\begin{array}{l}\text { Out- } \\
\text { degree }\end{array}$ & $\begin{array}{l}\text { In- } \\
\text { degree }\end{array}$ \\
\hline & & & Gesamt & & & Gesamt & & & Gesamt & & \\
\hline \multirow[t]{5}{*}{1} & 8 & 0 & 37 & 0,38 & 0,41 & 111 & 0,29 & 0,37 & 12 & 0,33 & 0,42 \\
\hline & 9 & 0 & & 0,05 & 0,11 & & 0,05 & 0,05 & & 0,08 & 0,08 \\
\hline & 16 & 0 & & 0,32 & 0,16 & & 0,41 & 0,34 & & 0,08 & 0,25 \\
\hline & 22 & 0 & & 0,08 & 0,19 & & 0,18 & 0,19 & & 0,50 & 0,25 \\
\hline & LK & - & & 0,16 & 0,14 & & 0,08 & 0,05 & & - & - \\
\hline \multirow[t]{5}{*}{2} & 5 & 1 & 82 & 0,16 & 0,37 & 82 & 0,17 & 0,33 & 92 & 0,42 & 0,39 \\
\hline & 7 & 1 & & 0,32 & 0,28 & & 0,41 & 0,22 & & 0,29 & 0,26 \\
\hline & 20 & 0 & & 0,05 & 0,04 & & 0,06 & 0,12 & & 0,08 & 0,09 \\
\hline & 23 & 1 & & 0,34 & 0,13 & & 0,20 & 0,20 & & 0,21 & 0,32 \\
\hline & LK & - & & 0,13 & 0,18 & & 0,15 & 0,13 & & - & - \\
\hline \multirow[t]{5}{*}{3} & 3 & 1 & 75 & 0,31 & 0,33 & 135 & 0,29 & 0,40 & 59 & 0,25 & 0,44 \\
\hline & 4 & 0 & & 0,08 & 0,31 & & 0,23 & 0,31 & & 0,22 & 0,24 \\
\hline & 13 & 0 & & 0,23 & 0,05 & & 0,18 & 0,06 & & 0,14 & 0,12 \\
\hline & 19 & 1 & & 0,19 & 0,19 & & 0,22 & 0,14 & & 0,20 & 0,39 \\
\hline & LK & - & & 0,20 & 0,12 & & 0,09 & 0,04 & & - & - \\
\hline \multirow[t]{5}{*}{4} & 10 & 0 & 79 & 0,19 & 0,19 & 92 & 0,28 & 0,16 & 81 & 0,36 & 0,30 \\
\hline & 11 & 1 & & 0,18 & 0,27 & & 0,23 & 0,17 & & 0,24 & 0,23 \\
\hline & 15 & 0 & & 0,18 & 0,03 & & 0,11 & 0,13 & & 0,18 & 0,34 \\
\hline & 25 & 1 & & 0,38 & 0,47 & & 0,30 & 0,45 & & 0,23 & 0,14 \\
\hline & LK & - & & 0,08 & 0,05 & & 0,08 & 0,09 & & - & - \\
\hline \multirow[t]{5}{*}{5} & 2 & 0 & 78 & 0,18 & 0,38 & 107 & 0,40 & 0,32 & 33 & 0,28 & 0,28 \\
\hline & 12 & 0 & & 0,09 & 0,04 & & 0,13 & 0,13 & & 0,52 & 0,38 \\
\hline & 14 & 0 & & 0,14 & 0,12 & & 0,05 & 0,13 & & 0,14 & 0,21 \\
\hline & 18 & 1 & & 0,44 & 0,35 & & 0,26 & 0,37 & & 0,07 & 0,14 \\
\hline & LK & - & & 0,16 & 0,13 & & 0,05 & 0,16 & & - & - \\
\hline
\end{tabular}

$N=20$

${ }^{a}$ Von der Lehrkraft eingeschätzte Note im Fach Deutsch: 0=leistungsschwächer (Note 3 und 4), 1 = leistungsstärker (Note 1 und 2)

größer der Durchmesser, desto häufiger sind die Akteur*innen in die Gruppenarbeit aktiv involviert (senden häufiger Redebeiträge und werden häufiger mit Redebeiträgen adressiert). Betrachtet man nun Abb. 3, so zeigt sich augenscheinlich, dass sich vereinzelte Schüler*innen nur peripher in ergebnisorientierte und prozessorientierte Interaktionen einbringen, aber zentral in Interaktionen zu nicht-aufgabenbezogenen Themen involviert sind, da sie häufiger einen nicht-aufgabenbezogenen Redebeitrag senden (S22, S5, S19, S10, S12). Einige Schüler*innen sind auf allen Ebenen der Auseinandersetzung nur peripher bis gar nicht aktiv an der Interaktion beteiligt (S9, S20, S13, S14). 
Tab. 4 Ergebnisse der Korrelationsanalyse zwischen der Leistungsgruppe und den empfangenen und gesendeten Redebeiträgen (In- bzw. Outdegree) spezifiziert nach der Art der Auseinandersetzung mit dem Lerngegenstand

\begin{tabular}{|c|c|c|c|c|c|c|c|c|}
\hline & & (1) & (2) & (3) & (4) & (5) & (6) & (7) \\
\hline (1) & Leistungsgruppe & 1,00 & - & - & - & - & - & - \\
\hline (2) & $\begin{array}{l}\text { Indegree Ergebnisori- } \\
\text { entiert }\end{array}$ & $0,53^{*}$ & 1,00 & - & - & - & - & - \\
\hline (3) & $\begin{array}{l}\text { Outdegree Ergebnis- } \\
\text { orientiert }\end{array}$ & $0,48^{*}$ & $0,52 *$ & 1,00 & - & - & - & - \\
\hline (4) & $\begin{array}{l}\text { Indegree Prozessori- } \\
\text { entiert }\end{array}$ & 0,28 & $0,62 * *$ & $0,62 * *$ & 1,00 & - & - & - \\
\hline (5) & $\begin{array}{l}\text { Outdegree Prozess- } \\
\text { orientiert }\end{array}$ & 0,39 & $0,64 * *$ & $0,86^{* *}$ & $0,65^{* *}$ & 1,00 & - & - \\
\hline (6) & $\begin{array}{l}\text { Indegree Nicht- } \\
\text { aufgabenbezogen }\end{array}$ & 0,19 & 0,15 & 0,20 & 0,25 & 0,27 & 1,00 & - \\
\hline (7) & $\begin{array}{l}\text { Outdegree Nicht- } \\
\text { aufgabenbezogen }\end{array}$ & $-0,01$ & $-0,20$ & 0,19 & 0,10 & 0,09 & $0,58 * *$ & 1,00 \\
\hline
\end{tabular}

$N=$ 20; Von der Lehrkraft eingeschätzte Note im Fach Deutsch: 0= leistungsschwächer (Note 3 und 4), $1=$ leistungsstärker (Note 1 und 2)

$* p<0,05 ; * * p<0,01$

Fragestellung 4 Um die Rollen der Schüler*innen inhaltlich interpretieren zu können, wurden die In- und Outdegree-Werte mit der personenspezifischen Eigenschaft „von der Lehrkraft eingeschätzte Fachnote" korreliert. Tab. 4 zeigt die Korrelationen zwischen der Leistungsgruppe (leistungsstärker: Note 1 und 2; leistungsschwächer: Note 3 und 4) und dem In- und Outdegree-Wert, spezifiziert nach der Ebene der Auseinandersetzung. Es zeigt sich, dass die Leistungsgruppe signifikant positiv mit den ergebnisorientierten Redebeiträgen (sowohl In- als auch Outdegree) korreliert. Schüler*innen, die als eher leistungsstärker von der Lehrkraft eingeschätzt wurden, senden demnach häufiger einen ergebnisorientierten Redebeitrag an ihre Gruppenmitglieder bzw. die Lehrkraft (nutzen das ihnen geboten Lernangebot intensiver) und werden auch häufiger mit einem ergebnisorientierten Redebeitrag adressiert. Leistungsstärkere Schüler*innen dieser Klasse nehmen folglich bezüglich der Ergebnisorientierung eine zentrale Stellung im Netzwerk ein: sie ermöglichen mehr Lernangebote, nutzen die ihnen ermöglichten Lernangebote aber auch häufiger und schaffen dadurch weitere Lernangebote für die Gruppenmitglieder.

Fragestellung 5 Tab. 5 zeigt die prozentuale Verteilung derjenigen Redebeiträge innerhalb des ko-konstruktiven Interaktionsprozesses, die Bezug auf einen vorherigen Redebeitrag nehmen oder keinen Bezug aufweisen. Es werden die prozentualen Werte für die individuellen Schüler*innen und die auf Gruppenebene aggregierten Werte gezeigt. Die Lehrkraft wurde dabei nicht berücksichtigt. Es wird deutlich, dass die Schüler*innen häufig Bezug aufeinander nehmen, wobei bei ergebnisorientierten Redebeiträgen häufiger Bezug aufeinander genommen wird als dies bei prozessorientierten Redebeiträgen der Fall ist. Auf Schüler*innenebene zeigt sich, dass manche Schüler*innen häufiger Redebeiträge ohne Bezug senden als dass sie Bezug aufeinander nehmen (z. B. S16, S7, S3, S14). 

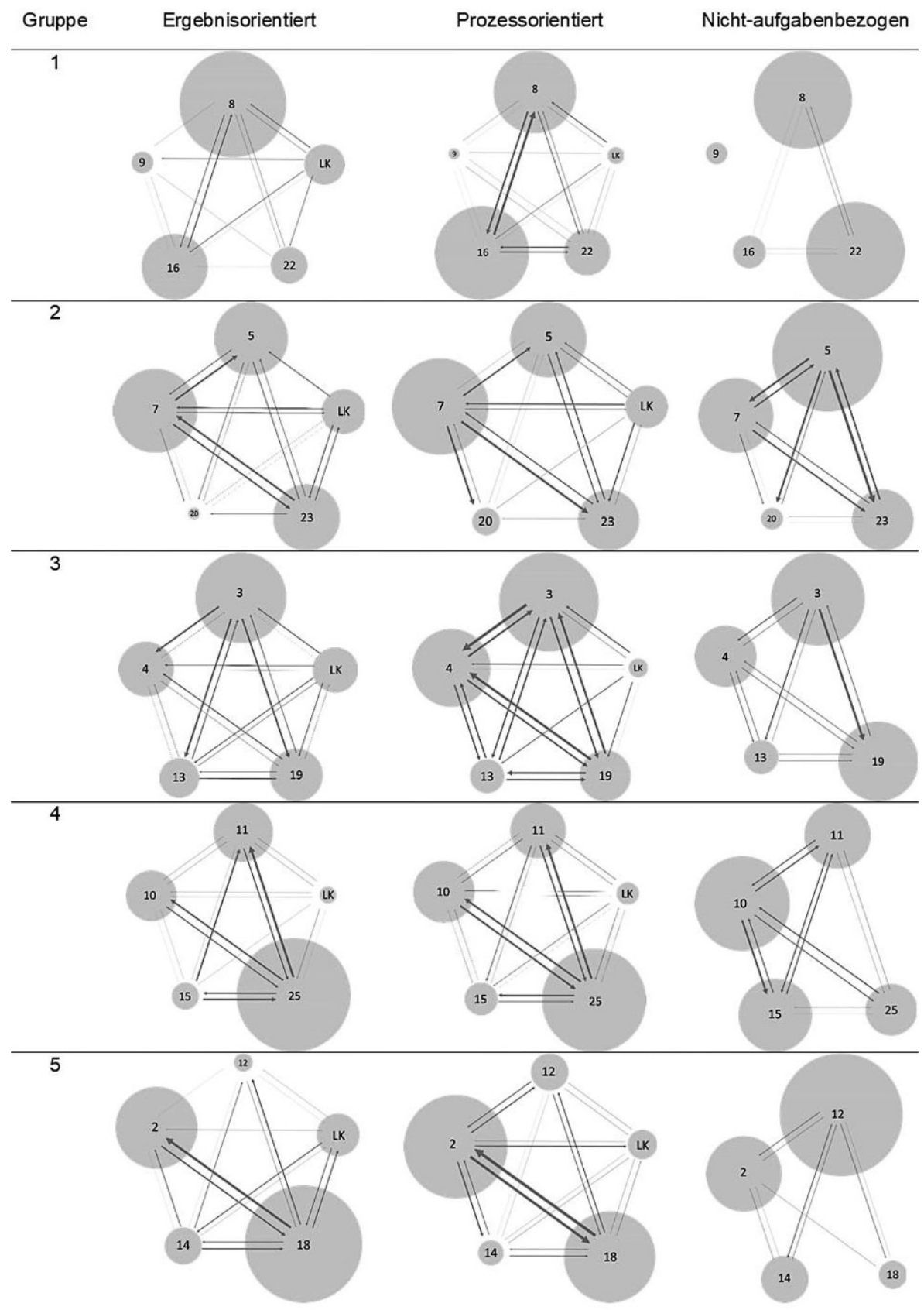

Abb. 3 Anzahl spezifischer Interaktionen zwischen den Schüler*innen und der Lehrkraft $(L K)$ in den jeweiligen Gruppen 
Tab. 5 Prozentuale Verteilung der Redebeiträge der Schüler*innen auf Individual- und Gruppenebene, die einen Bezug oder keinen Bezug zu einem vorherigen Beitrag aufwiesen

\begin{tabular}{|c|c|c|c|c|c|c|c|}
\hline \multicolumn{4}{|c|}{ Gruppe Schüler*in Ergebnisorientiert } & \multicolumn{2}{|c|}{ Prozessorientiert } & \multicolumn{2}{|c|}{ Nicht-aufgabenbezogen } \\
\hline & & $\begin{array}{l}\text { Ohne } \\
\text { Bezug }\end{array}$ & $\begin{array}{l}\text { Mit Be- } \\
\text { zug }\end{array}$ & $\begin{array}{l}\text { Ohne } \\
\text { Bezug }\end{array}$ & $\begin{array}{l}\text { Mit Be- } \\
\text { zug }\end{array}$ & $\begin{array}{l}\text { Ohne } \\
\text { Bezug }\end{array}$ & Mit Bezug \\
\hline \multirow[t]{5}{*}{1} & 8 & 40,00 & 46,15 & 30,43 & 31,65 & 28,57 & 40,00 \\
\hline & 9 & 0,00 & 7,69 & 4,35 & 5,06 & 14,29 & 0,00 \\
\hline & 16 & 60,00 & 34,62 & 30,43 & 48,10 & 0,00 & 20,00 \\
\hline & 22 & 0,00 & 11,54 & 34,78 & 15,19 & 57,14 & 40,00 \\
\hline & Gruppe & 16,13 & 83,87 & 22,55 & 77,45 & 58,33 & 41,67 \\
\hline \multirow[t]{5}{*}{2} & 5 & 20,00 & 18,03 & 17,65 & 22,22 & 72,73 & 32,86 \\
\hline & 7 & 70,00 & 31,15 & 55,88 & 41,67 & 22,73 & 31,43 \\
\hline & 20 & 0,00 & 6,56 & 11,76 & 2,78 & 0,00 & 10,00 \\
\hline & 23 & 10,00 & 44,26 & 14,71 & 33,33 & 4,55 & 25,71 \\
\hline & Gruppe & 14,08 & 85,92 & 48,57 & 51,43 & 23,91 & 76,09 \\
\hline \multirow[t]{5}{*}{3} & 3 & 45,45 & 36,73 & 47,37 & 18,18 & 60,71 & 29,03 \\
\hline & 4 & 0,00 & 12,24 & 12,28 & 36,36 & 10,71 & 32,26 \\
\hline & 13 & 18,18 & 30,61 & 12,28 & 25,76 & 25,00 & 3,23 \\
\hline & 19 & 36,36 & 20,41 & 28,07 & 19,70 & 3,57 & 35,48 \\
\hline & Gruppe & 18,33 & 81,67 & 46,34 & 53,66 & 47,46 & 52,54 \\
\hline \multirow[t]{5}{*}{4} & 10 & 20,00 & 20,63 & 5,88 & 25,86 & 41,94 & 34,00 \\
\hline & 11 & 30,00 & 17,46 & 17,65 & 31,03 & 22,58 & 24,00 \\
\hline & 15 & 10,00 & 20,63 & 5,88 & 15,52 & 12,90 & 20,00 \\
\hline & 25 & 40,00 & 41,27 & 70,59 & 27,59 & 22,58 & 22,00 \\
\hline & Gruppe & 13,70 & 86,30 & 22,67 & 77,33 & 38,27 & 61,73 \\
\hline \multirow[t]{5}{*}{5} & 2 & 27,78 & 19,15 & 17,24 & 39,73 & 16,67 & 33,33 \\
\hline & 12 & 0,00 & 14,89 & 20,69 & 10,96 & 61,11 & 26,67 \\
\hline & 14 & 22,22 & 14,89 & 27,59 & 8,22 & 22,22 & 26,67 \\
\hline & 18 & 50,00 & 51,06 & 34,48 & 41,10 & 0,00 & 13,33 \\
\hline & Gruppe & 27,69 & 72,31 & 28,43 & 71,57 & 54,55 & 45,45 \\
\hline
\end{tabular}

$N=20$

Fragestellung 6 Um die Entwicklung der dynamischen Interaktionsprozesse zu beschreiben, wurden in einem ersten Schritt zeitabhängige Visualisierungen der Interaktionen in den einzelnen Gruppen erstellt. Diese ermöglichen es, im zeitlichen Verlauf des Unterrichts die Aktivität der einzelnen Schüler*innen zu betrachten und unterschiedliche Phasen in deren Interaktionsprozess herauszuarbeiten. Im zusätzlichen Onlinematerial sind für jede der fünf Gruppen sowie die gesamte Klasse Animationen bereitgestellt, in welchen der ko-konstruktive Interaktionsprozess bzw. die gerichteten Redebeiträge der Schüler*innen und der Lehrkraft im zeitlichen Verlauf des Unterrichts ersichtlich werden (Onlinematerial 2 bis 8). Die Animationen verdeutlichen den dynamischen Prozess ko-konstruktiver Interaktionsprozesse. Abb. 4 zeigt zudem den in den Animationen zu beobachtenden Verlauf der dyadischen Redebeiträge sowie deren Gesprächsinhalt über die Gruppenarbeitsphase hinweg in einem Verlaufsdiagramm. Eine augenscheinliche Analyse der Animationen sowie der Abb. 4 zeigt, dass die Interaktion zwischen den Schüler*innen in Gruppe 5 


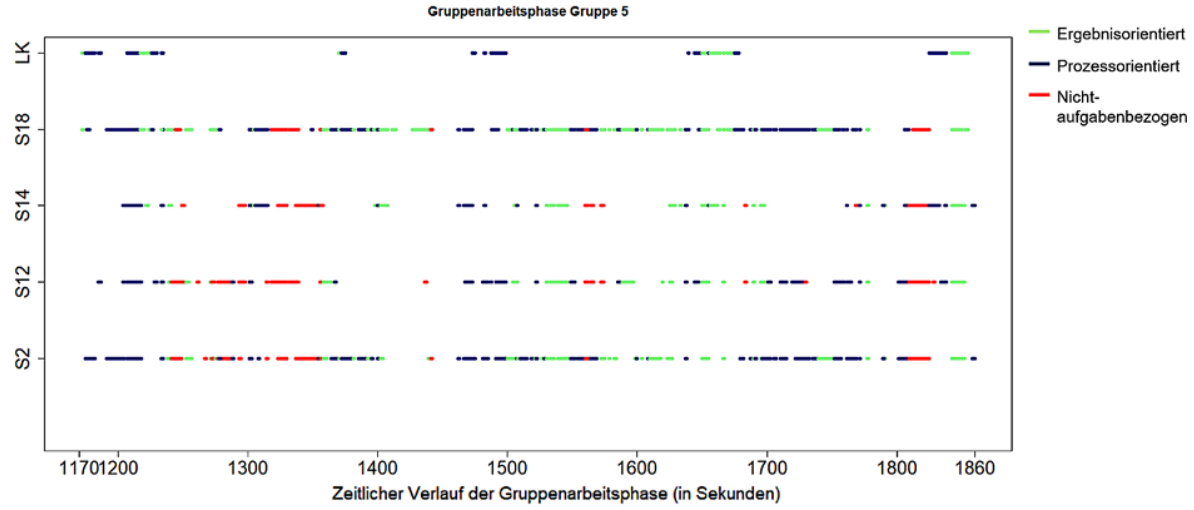

Abb. 4 Entwicklung des Interaktionsprozesses über die gesamte Gruppenarbeitsphase hinweg

beispielsweise $\mathrm{zu}$ Beginn eher prozessorientiert ist. Es folgt eine nicht-aufgabenbezogene Phase, in der alle Schüler*innen beteiligt sind. Daran schließt sich eine längere Arbeitsphase an, in der die Schüler*innen aufgabenbezogen miteinander interagieren, wobei überwiegend Schüler*in 18 und Schüler*in 2 miteinander arbeiten. Am Ende der Gruppenarbeitsphase findet erneut eine nicht-aufgabenbezogene Interaktionsphase statt. Die Lehrkraft ist zu fünf Zeitpunkten am Gruppentisch in den Interaktionsprozess involviert. Augenscheinlich zeigt sich, dass während und kurz nachdem die Lehrkraft am Gruppentisch ist, die Schüler*innen überwiegend aufgabenbezogen, ergebnisorientiert interagieren. Die Abbildung der Interaktionsprozesse im zeitlichen Verlauf der Gruppenarbeitsphase ermöglicht es, die Entwicklungen der aktiven Beteiligung der individuellen Schüler*innen sowie der gesamten Gruppe zu identifizieren. Im zusätzlichen Onlinematerial können die Entwicklungen des Interaktionsprozesses für die anderen vier Gruppen eingesehen werden (Onlinematerial 9). Die Animationen ermöglichen es, die Komplexität der gleichzeitig ablaufenden Interaktionsprozesse im Unterricht darzustellen.

\section{Diskussion}

Unterrichtsprozesse finden überwiegend in sozialen Interaktionen zwischen Lehrkräften und Schüler*innen sowie zwischen den Schüler*innen untereinander statt (Fend 2019; Vieluf et al. 2020). Dabei gestalten sie gemeinsam in der Interaktion kokonstruktiv ein Lernangebot, welches von den Schüler*innen wahrgenommen und genutzt werden kann (Fend 2008; Helmke 2009; Lipowsky 2020; Seidel 2014; Vieluf et al. 2020). Die aktive Beteiligung der Schüler*innen an der Interaktion kann dabei als Resultat bzw. Indikator der individuellen Nutzungsprozesse betrachtet werden. In der sozialen Interaktion äußern sich diese überwiegend in Form von verbalen Redebeiträgen, die die Schüler*innen an andere, an der Interaktion Beteiligte, senden. Diese Beteiligung stellt dabei wiederum für andere Schüler*innen ein potenzielles Lernangebot dar, welches diese ebenso wahrnehmen und nutzen können. Um die Nutzungsprozesse zu erfassen, könnte die Analyse der ko-konstruktiven Interakti- 
onsprozesse ein gewinnbringender Zugang sein. Um die (differenzielle) Beteiligung an ko-konstruktiven Interaktionsprozessen sowie die Ausgestaltung der Interaktionsprozesse im Detail verstehen und analysieren zu können, sind jedoch methodische Ansätze notwendig, die zum einen erfassen, wer wie stark an der Bearbeitung einer Lernaufgabe beteiligt ist und wer konkret ein Lernangebot (hier: operationalisiert als empfangener, aufgabenbezogener, ergebnisorientierter Redebeitrag) erhält. Zum anderen sollte erfasst werden, wer mit wem gemeinsam ko-konstruktiv an der Lösungsfindung beteiligt ist (Interaktion zwischen Akteur*innen) und wie sich die Beteiligung über die Zeit verändert (dynamische Entwicklung des ko-konstruktiven Interaktionsprozesses). Im Rahmen des Artikels wurde die Netzwerkanalyse als methodischer Ansatz vorgestellt, um derartige dynamische Interaktionsprozesse beschreiben und analysieren zu können.

\subsection{Zusammenfassung und Diskussion der Ergebnisse}

Anhand von exemplarischen inhaltlichen Ergebnissen wurde das Potenzial der Methode aufgezeigt und die übergreifende Fragestellung, inwieweit durch Netzwerkanalysen konstituierende Merkmale ko-konstruktiver Interaktionsprozesse im Unterricht beschrieben und analysiert werden können, bearbeitet. Die Netzwerkanalyse ermöglicht es, aufeinander bezogenen Redebeiträge im Unterricht zusammenhängend und komplex abzubilden. Eine analytische Annäherung an die entstehenden Netzwerke über Knotengrade und Verlaufsdiagramme zeigt, wie es gelingen kann, die einzelnen Rollen und Stellungen der Akteur*innen in der Interaktion zu identifizieren und erste Strukturen der Interaktionsprozesse zu visualisieren.

Die Korrelation der In- und Outdegree-Werte mit dem hier beispielhaft genutzten Hintergrundmerkmal „Fachnote“ ermöglicht es, die Systematik des Netzwerks (also wie intensiv die Schüler*innen im Interaktionsprozess eingebunden sind) anfänglich zu ergründen. In der Anwendung der Methode auf größere Stichproben könnten neben dem Leistungsniveau der Schüler*innen auch weitere Hintergrundmerkmale, die die Beteiligung der Schüler*innen an der Interaktion beeinflussen, berücksichtigt werden. Hierzu zählen beispielsweise das Interesse am Fach (Nemeth et al. 2019), das Sprachniveau der Schüler*innen, der sozioökonomische Status sowie ein potenzieller Migrationshintergrund (Decristan et al. 2019). Auch das Vorwissen (Decristan et al. 2019) könnte die aktive Beteiligung begünstigen. Im Sinne einer soziologischen Betrachtung können auch Rollenbilder oder verschiedene weitere soziale Beziehung zwischen den Akteur*innen (z. B. Freundschaften oder Vorerfahrungen in der Zusammenarbeit) die Stellung der Akteur*innen im Netzwerk begründen (Zander et al. 2017). So könnten Analysen, die auf das Beschreiben und Verstehen von Interaktionsprozessen im Unterricht abzielen, ebenso von einem systemtheoretischen Blick auf das Unterrichtsgeschehen profitieren und die zugrundeliegenden Netzwerke auf Multiplexität, also die Überlappung mehrerer Rollen und Merkmale der interagierenden Schüler*innen und Lehrkräfte, prüfen.

Mit der Berechnung eines Bezugsindizes (in Anlehnung an Nemeth et al. 2019) konnte in diesen exemplarischen Analysen aufgezeigt werden, dass manche Schüler*innen häufiger als andere mit ihrem Beitrag Bezug aufeinander nehmen. Dies stellt eine erste Annäherung an die Analyse der ko-konstruktiven Wissensbildung im 
Unterricht dar. Dabei bleibt jedoch noch ungeklärt, ob die Redebeiträge, die keinen Bezug aufweisen, Redebeiträge mit neuen Diskussionsthemen sind, die die Schüler*innen in der Lösung des Problems weiterbringen, oder ob Schüler*innen Redebeiträge andere Schüler*innen ignorieren. In anderen Worten ausgedrückt: findet wirklich ein qualitativ hochwertiger Austausch zwischen den Schüler*innen statt? Hier könnte die qualitative Analyse der Redebeiträge der Schüler*innen eine fruchtbare Ergänzung sein. Die Kategoriensysteme von Ranger (2017) oder Hennessy et al. (2016) bieten hierfür mögliche Anknüpfungspunkte.

Grundsätzlich gilt: Werden Interaktionsprozesse im Unterricht netzwerkanalytisch ausgewertet, entsteht ein Ereignisnetzwerk, welches durch sich schnell verändernde Ereignisse zwischen den einzelnen Akteur*innen des Netzwerks gekennzeichnet ist. Ereignisnetzwerke sehen je Messzeitpunkt sehr verschieden aus, weshalb eine analytische Annäherung über zeitaggregierte, statische Netzwerke erfolgt. Um die dynamische Entwicklung des ko-konstruktiven Interaktionsprozesses im Unterricht zwischen Schüler*innen jedoch analysieren zu können, wurden im Rahmen dieses Artikels Animationen der kodierten Interaktionen sowie Verlaufsdiagramme vorgestellt. Beide Vorgehensweisen ermöglichen es, den Interaktionsprozess während der Gruppenarbeit in unterschiedliche Phasen (hier exemplarisch bezüglich der Kategorien eher aufgabenorientierte oder nicht-aufgabeorientierte Phasen der Interaktion, Interaktionsphasen mit der Lehrkraft) einzuteilen. In den beispielhaften Ergebnissen zeigt sich im Verlauf, dass die Schüler*innen aktivere (aufgabenbezogene) und weniger aktive Phasen durchleben. Auch zeigt sich, dass manche Schüler*innen eher bei nicht-aufgabenbezogenen Phasen in die Interaktion involviert sind und andere Schüler*innen eher in aufgabenorientierten Phasen sich aktiv einbringen. Besonders die Animationen verdeutlichten die Komplexität von ko-konstruktiven Interaktionsprozessen im Unterricht, da diese die gleichzeitig ablaufenden Ereignisse (Sprecher*innenwechsel, Parallelgespräche) anschaulich dokumentieren. Verlaufsdiagramme reduzieren hingegen die Komplexität, sodass Interaktionsprozesse, die parallel stattfinden, nicht adäquat abgebildet werden können.

Bezugnehmend auf die exemplarischen Ergebnisse dieser Studie wird der Prozesscharakter von Angebot und Nutzung besonders hervorgehoben. Angebot und Nutzung stehen in einer dynamischen Wechselbeziehung zueinander: Schüler*innen, die in dieser Stichprobe häufiger ein Lernangebot nutzen und dadurch für andere ein Lernangebot ermöglichen, werden auch häufiger wieder mit einem potenziellen Lernangebot adressiert. In einem nächsten Schritt könnte dies in größer angelegten Folgestudien weiter untersucht werden. Dabei wäre eine netzwerkanalytische Betrachtung der Daten dahingehend gewinnbringend, dass analysiert werden könnte, welche Schüler*innen (mit welchen Hintergrundmerkmalen) mit welchen Schüler*innen häufiger interagieren (also beispielsweise: senden leistungsstarke Schüler*innen häufiger auch an ihresgleichen einen Redebeitrag, werden alle Schüler*innen gleichermaßen in die Interaktion eingebunden oder welche Schüler*innen reagieren häufiger auf ein potenzielles Lernangebot?). Eine fruchtbare Erkenntnis könnte folglich auch die Analyse der Redebeiträge bezüglich ihrer Qualität liefern: wer sind die Schüler*innen, die im Sinne eines lernförderlichen ko-konstruktiven Interaktionsprozesses Ideen anderer Schüler*innen kritisch hinterfragen, Widersprüche aufklären, neue Ideen einbringen und/oder Problemlösungen falsifi- 
zieren bzw. verifizieren (Lipowsky 2021; Ranger 2017)? Hierfür könnten Ansätze der Diskursanalyse mit klassischen Netzwerkanalysen (z. B. Bruun et al. 2019; Ryu und Lombardi 2015) kombiniert werden, um tiefere Einblicke in die Entwicklung der Interaktionsprozesse und die Qualität dieser zu erhalten. Auch könnte dies zu einem tieferen Verständnis des situationalen Lernangebots, welches in der Interaktion der Schüler*innen mit der Lehrkraft oder anderen Schüler*innen entsteht, beitragen (Seidel 2020).

\subsection{Limitationen}

Die Studie zielte darauf ab, das Potenzial netzwerkanalytischer Ansätze zur Beschreibung und Analyse von Videodaten beispielhaft aufzuzeigen. Mit diesem Vorgehen sind jedoch einige Limitationen verbunden.

Die netzwerkbasierte Analyse von ko-konstruktiven Interaktionsprozessen im Unterricht verlangt es, Redebeiträge im Unterricht im Detail und kleinschrittig zu kodieren. Die detaillierte Kodierung und Beschreibung, wie sie in dieser exemplarischen Studie vorgestellt wurde, ist lediglich aufgrund eines schüler*innenzentrierten Aufnahmesystems möglich (Troll et al. 2020). Klassische Videostudien verwenden meist ein Aufnahmesystem mit zwei fest installierten Kameras (dynamische Lehrkraftkamera und Überblickskamera; Seidel et al. 2003), über welche die Interaktionsprozesse zwischen der Lehrkraft und den Schüler*innen, nicht aber zwischen den Schüler*innen im Detail beobachtet werden können. Die detaillierte Kodierung der Interaktionsprozesse auf Ebene der Schüler*innen mittels eines schüler*innenzentrierten Aufnahmesystems, mit vielen im Klassenzimmer installierten Kameras, stellt sich als sehr arbeitsaufwändig heraus, was bei zukünftigen Studien zu berücksichtigen gilt. Dies könnte auch ein Grund für die lediglich gute Beurteiler*innenübereinstimmung bzgl. der Art des Redebeitrags sein. Obwohl die Kodierer*innen intensiv geschult wurden, konnte nur eine gute Übereinstimmung von Cohen's Kappa zwischen k=0,63 und k=0,69 in der Klassifizierung der Art des Redebeitrags erzielt werden. Auch gilt es zu beachten, dass in kollaborativen Gruppenarbeitsphasen, wie es in den Beispieldaten dieser Studie der Fall ist, die Schüler*innen im ständigen Wechsel miteinander sprechen - verdeutlicht wird dies auch anhand der Animationen im zusätzlichen Onlinematerial - wodurch eine detaillierte Kodierung der einzelnen Redebeiträge erschwert werden kann.

Bezüglich der Operationalisierung des Konstrukts „Nutzung“ ist an dieser Stelle eine kritische Betrachtung der Redebeiträge als Indikator bzw. Resultat mentaler Nutzungsprozesse (Seidel 2020; Vieluf et al. 2020) zu diskutieren. Schüler*innen, die sich in die Interaktion nicht verbal einbringen, nutzen in dieser Definition das Lernangebot auch nicht intensiv. Dennoch können Schüler*innen, die sich nicht aktiv beteiligen, trotzdem dem Unterricht gedanklich aufmerksam folgen und das Lernangebot demnach nutzen (O'Connor et al. 2017; Renkl 2011). Studien haben dahingehend bereits gezeigt, dass auch stille Schüler*innen von der Interaktion anderer profitieren (Pauli und Lipowsky 2007), was bedeutet, dass nicht nur die aktive Beteiligung an der Interaktion den Lernprozess anregen kann. Diskutiert werden müsste dann aber auch, wie die Nutzung, welche hier ein Prozessmerkmal des Unterrichts (Vieluf et al. 2020) darstellt, zusätzlich erfasst werden kann, ohne dass 
der natürliche Verlauf des Interaktionsprozesses, beispielsweise über kleine Fragebogenerhebungen während des Unterrichts in Sinne eines Experience Sampling Verfahren, gestört werden würde. Die Beobachtung als Datenerhebungsverfahren ermöglicht es lediglich, die sichtbaren Aktivitäten der Akteur*innen im Unterricht zu erfassen und zu interpretieren.

Ein weiterer Kritikpunkt an dieser Stelle ist, dass in den Analysen die Rolle der Lehrkraft bisher kaum Berücksichtigung fand. Da die exemplarische Studie das Potenzial des dargestellten methodischen Ansatzes beispielhaft herauszuarbeiten versuchte, wurde die Rolle der Lehrkraft vernachlässigt; auch wenn den Autor*innen bewusst ist, dass der Lehrkraft im Sinne des sozial-konstruktivistischen Lehr-LernVerständnisses eine bedeutende Rolle zukommt (Howe 2013). In Interaktionsprozessen nimmt die Lehrkraft als kompetentere*r Gesprächspartner*in eine unterstützende Funktion ein, indem sie den Schüler*innen in deren Zone der nächsten Entwicklung Lernangebote ermöglicht (Herrmann et al. 2021; Howe 2013; van Braak et al. 2021; van de Pol et al. 2010). Ein wichtiges Instrument der Lehrkräfte sind dabei sogenannte Scaffolding-Strategien, die für die Schüler*innen ein Lerngerüst (,scaffold“) bereitstellen, das deren Lernprozess strukturiert sowie zum weiteren Denken angeregt und zur Bewältigung einer Aufgabe befähigt (Herrmann et al. 2021; van Braak et al. 2021; van de Pol et al. 2010). Die Rolle der Lehrkraft während des Unterrichts ist daher u.a. eher die einer ,,adaptiven Lernberaterin“, um situationssensibel die Zusammenarbeit der Schüler*innen zu unterstützen und den sachbezogenen Lerndialog zwischen den Gruppenmitgliedern zu intensivieren (Pauli und Reusser 2000, S. 431). Die Redebeiträge der Lehrkraft erfüllen demnach einen anderen Zweck als die der Schüler*innen und könnten daher in zukünftigen Studien auch dahingehend erfasst und interpretiert werden.

\subsection{Fazit}

Zusammenfassend lässt sich festhalten, dass die Netzwerkanalyse videobasierter Daten zur Beschreibung der Wechselwirkung von Angebot und Nutzung im Klassenraum ein gewinnbringender Ansatzpunkt für die zukünftige Analyse von Interaktionsprozessen sein könnte, welcher sich nun in weiteren empirischen Arbeiten unter Beweis stellen muss. Es können Schlussfolgerungen und Interpretationen über das Interaktionsverhalten der Akteur*innen, die mit gewöhnlichen Analysetechniken gewonnen wurden, bestätigt und weiter kontextualisiert werden. Außerdem können netzwerkanalytische Studien um Fragestellungen, welche auf die Auswirkungen der ko-konstruktiven Interaktionsprozesse abzielen, ergänzt werden. Beispielsweise könnten sie genutzt werden, um die differenziellen Auswirkungen der Interaktionsprozesse auf die Leistungsentwicklung der Schüler*innen zu analysieren. Erkenntnisse aus derart angelegten Studien könnten zu einem tieferen Verständnis von kokonstruktiver Wissensbildung beitragen, die wiederum den Lernerfolg der Schüler*innen beeinflusst. Ein genaues Verständnis dafür, welche Prozesse innerhalb eines Netzwerks ablaufen, welche Einflussfaktoren diese erklären könnten und wie die individuellen und vielfältigen Profile der Schüler*innen damit zusammenhängen, könnte demnach zukünftig einen Mehrwert darstellen, um die individuellen 
Nutzungsprozesse der Schüler*innen und folglich auch deren Lernerfolg weiter zu erklären.

Zusatzmaterial online Zusätzliche Informationen sind in der Online-Version dieses Artikels (https://doi. org/10.1007/s42010-022-00142-1) enthalten.

Funding Open Access funding enabled and organized by Projekt DEAL.

Open Access Dieser Artikel wird unter der Creative Commons Namensnennung 4.0 International Lizenz veröffentlicht, welche die Nutzung, Vervielfältigung, Bearbeitung, Verbreitung und Wiedergabe in jeglichem Medium und Format erlaubt, sofern Sie den/die ursprünglichen Autor(en) und die Quelle ordnungsgemäß nennen, einen Link zur Creative Commons Lizenz beifügen und angeben, ob Änderungen vorgenommen wurden.

Die in diesem Artikel enthaltenen Bilder und sonstiges Drittmaterial unterliegen ebenfalls der genannten Creative Commons Lizenz, sofern sich aus der Abbildungslegende nichts anderes ergibt. Sofern das betreffende Material nicht unter der genannten Creative Commons Lizenz steht und die betreffende Handlung nicht nach gesetzlichen Vorschriften erlaubt ist, ist für die oben aufgeführten Weiterverwendungen des Materials die Einwilligung des jeweiligen Rechteinhabers einzuholen.

Weitere Details zur Lizenz entnehmen Sie bitte der Lizenzinformation auf http://creativecommons.org/ licenses/by/4.0/deed.de.

\section{Literatur}

Ackermann, S. (2011). Klassengespräch im Mathematikunterricht: Eine Pilotstudie im Rahmen des Projekts „Persönlichkeits- und Lernentwicklung von Grundschulkindern“. Studium und Forschung, Bd. 19. Kassel: Univ. Press.

Battistich, V., Solomon, D., \& Delucchi, K. (1993). Interaction processes and student outcomes in cooperative learning groups. The Elementary School Journal, 94(1), 19-32. https://doi.org/10.1086/461748.

Bender-deMoll, S., \& Morris, M. (2019). ndtv: Network DynamicTemporal Visualizations. https://github. com/statnet/ndtv (Version 0.13.0) [Computer software]. CRAN. Zugegriffen: 19. Juli 2021

Böheim, R., Urdan, T., Knogler, M., \& Seidel, T. (2020). Student hand-raising as an indicator of behavioral engagement and its role in classroom learning. Contemporary Educational Psychology, 62, 101894.

Bokhove, C. (2018). Exploring classroom interaction with dynamic social network analysis. International Journal of Research \& Method in Education, 41(1), 17-37. https://doi.org/10.1080/1743727X.2016. 1192116.

van Braak, M., van de Pol, J., Poorthuis, A. M., \& Mainhard, T. (2021). A micro-perspective on students' behavioral engagement in the context of teachers' instructional support during seatwork: Sources of variability and the role of teacher adaptive support. Contemporary Educational Psychology, 64, 101928. https://doi.org/10.1016/j.cedpsych.2020.101928.

Bruun, J., Lindahl, M., \& Linder, C. (2019). Network analysis and qualitative discourse analysis of a classroom group discussion. International Journal of Research \& Method in Education, 42(3), 317-339. https://doi.org/10.1080/1743727X.2018.1496414.

Butts, C. T. (2008). Social network analysis: a methodological introduction. Asian Journal Of Social Psychology, 11(1), 13-41. https://doi.org/10.1111/j.1467-839X.2007.00241.x.

Decristan, J., Fauth, B., Heide, E.L., Locher, F. M., Troll, B., Kurucz, C., \& Kunter, M. (2019). Individuelle Beteiligung am Unterrichtsgespräch in Grundschulklassen: Wer ist (nicht) beteiligt und welche Konsequenzen hat das für den Lernerfolg? Zeitschrift für Pädagogische Psychologie. https://doi.org/ $10.1024 / 1010-0652 / \mathrm{a} 000251$.

Döring, N., \& Bortz, J. (2016). Forschungsmethoden und Evaluation in den Sozial- und Humanwissenschaften (5. Aufl.). Berlin, Heidelberg: Springer. https://doi.org/10.1007/978-3-642-41089-5.

Fend, H. (2008). Neue Theorie der Schule: Einführung in das Verstehen von Bildungssystemen (2. Aufl.). Wiesbaden: VS. https://doi.org/10.1007/978-3-531-91788-7.

Fend, H. (2019). Erklärungen von Unterrichtserträgen im Rahmen des Angebot-Nutzungs-Modells. In U. Steffens \& R. Messner (Hrsg.), Beiträge zur Schulentwicklung. Unterrichtsqualität: Konzepte und Bilanzen gelingenden Lehrens und Lernens. Grundlagen der Qualität von Schule (Bd. 3, S. 91-104). Münster, New York: Waxmann. 
Froehlich, D. E., van Waes, S., \& Schäfer, H. (2020). Linking quantitative and qualitative network approaches: a review of mixed methods social network analysis in education research. Review of Research in Education, 44(1), 244-268. https://doi.org/10.3102/0091732X20903311.

Fuhse, J. (Hrsg.). (2018). Soziale Netzwerke: Konzepte und Forschungsmethoden (2. Aufl.). Konstanz: UVK.

Grunspan, D.Z., Wiggins, B. L., \& Goodreau, S. M. (2014). Understanding classrooms through social network analysis: a primer for social network analysis in education research. CBE life sciences education, 13(2), 167-179. https://doi.org/10.1187/cbe.13-08-0162.

Handcock, M. S., Hunter, D. R., Butts, C.T., Goodreau, S. M., \& Morris, M. (2008). statnet: software tools for the representation, visualization, analysis and simulation of network data. Journal of statistical software, 24(1), 1548-7660. https://doi.org/10.18637/jss.v024.i01.

Haythornthwaite, C. (2001). Exploring multiplexity: social network structures in a computer-supported distance learning class. The Information Society, 17(3), 211-226. https://doi.org/10.1080/ 01972240152493065 .

Helmke, A. (Hrsg.). (2009). Unterrichtsqualität und Lehrerprofessionalität: Diagnose, Evaluation und Verbesserung des Unterrichts (3. Aufl.). Unterricht verbessern - Schule entwickeln. Seelze-Velber: Klett.

Hennessy, S., Rojas-Drummond, S., Higham, R., Márquez, A. M., Maine, F., Ríos, R. M., García-Carrión, R., Torreblanca, O., \& Barrera, M.J. (2016). Developing a coding scheme for analysing classroom dialogue across educational contexts. Learning, Culture and Social Interaction, 9, 16-44. https://doi. org/10.1016/j.lcsi.2015.12.001.

Herrmann, A., Bürgermeister, A., Lange-Schubert, K., \& Saalbach, H. (2021). Die Bedeutung von Partizipation und Scaffolding für die Leistung im naturwissenschaftlichen Sachunterricht in Klassen mit hohem und niedrigem Anteil mehrsprachiger Schüler*innen. Zeitschrift für Grundschulforschung. https://doi.org/10.1007/s42278-021-00112-z.

Hijzen, D., Boekaerts, M., \& Vedder, P. (2007). Exploring the links between students' engagement in cooperative learning, their goal preferences and appraisals of instructional conditions in the classroom. Learning and Instruction, 17(6), 673-687. https://doi.org/10.1016/j.learninstruc.2007.09.020.

Hofer, M. (1997). Lehrer-Schüler-Interaktion: 5. Kapitel. In F.E. Weinert (Hrsg.), Psychologie des Unterrichts und der Schule. Enzyklopädie der Psychologie Praxisgebiete Pädagogische Psychologie, (Bd. 3, S. 213-252). Göttingen: Hogrefe.

Howe, C. (2013). Scaffolding in context: peer interaction and abstract learning. Learning, Culture and Social Interaction, 2(1), 3-10. https://doi.org/10.1016/j.lcsi.2012.12.005.

Jurik, V., Gröschner, A., \& Seidel, T. (2013). How student characteristics affect girls' and boys' verbal engagement in physics instruction. Learning and Instruction, 23, 33-42. https://doi.org/10.1016/j. learninstruc.2012.09.002.

Klieme, E. (2006). Empirische Unterrichtsforschung: aktuelle Entwicklungen, theoretische Grundlagen und fachspezifische Befunde. Einführung in den Thementeil. Zeitschrift für Pädagogik, 52(6), 765-773.

de Laat, M., Lally, V., Lipponen, L., \& Simons, R.-J. (2007). Investigating patterns of interaction in networked learning and computer-supported collaborative learning: a role for Social Network Analysis. International Journal of Computer-Supported Collaborative Learning, 2(1), 87-103. https://doi.org/ 10.1007/s11412-007-9006-4.

Li, L., Shouhui, Z., \& Xinying, C. (2011). Beyond research: classroom interaction analysis techniques for classroom teachers. In 4th Redesigning Pedagogy International Conference. Singapore.

Lipowsky, F. (2020). Unterricht. In E. Wild \& J. Möller (Hrsg.), Pädagogische Psychologie (3. Aufl. S. 69-118). Berlin, Heidelberg: Springer. https://doi.org/10.1007/978-3-662-61403-7_4.

Lipowsky, F. (2021). Klassengespräche und Interaktionen im Unterricht. http://www.frank-lipowsky.de/ publikationen-vortraege/downloads/vortrag-leuphana-universitaet-lueneburg-2021/ Leuphana Universität Lüneburg, Lüneburg. Zugegriffen: 21. Okt. 2021

Mejeh, M.P., \& Hascher, T. (2021). Soziale Netzwerkanalyse als Erfassungsinstrument sozialer Interaktionen in der Schule. In G. Hagenauer \& D. Raufelder (Hrsg.), Soziale Eingebundenheit: Sozialbeziehungen im Fokus von Schule und LehrerInnenbildung (S. 33-45). Münster, New York: Waxmann.

Mercer, N., \& Dawes, L. (2014). The study of talk between teachers and students, from the 1970s until the 2010s. Oxford Review of Education, 40(4), 430-445. https://doi.org/10.1080/03054985.2014.934087.

Nemeth, L., Denn, A.-K., Hirstein, A., \& Lipowsky, F. (2019). Interaktionen von SchülerInnen in kooperativen Lernsituationen. In K. Verrière \& L. Schäfer (Hrsg.), Interaktion im Klassenzimmer: Forschungsgeleitete Einblicke in das Geschehen im Unterricht (S. 51-73). Wiesbaden: Springer VS. 
O'Connor, C., Michaels, S., Chapin, S., \& Harbaugh, A. G. (2017). The silent and the vocal: participation and learning in whole-class discussion. Learning and Instruction, 48, 5-13. https://doi.org/10.1016/ j.learninstruc.2016.11.003.

Pauli, C. (2006). Klassengespräch. In I. Hugener, C. Pauli \& K. Reusser (Hrsg.), Dokumentation der Erhebungs- und Auswertungsinstrumente zur schweizerisch-deutschen Videostudie „Unterrichtsqualität, Lernverhalten und mathematisches Verständnis“. Teil 3. Videoanalysen. Materialien zur Bildungsforschung, (Bd. 15, S. 124-147). GFPF.

Pauli, C., \& Lipowsky, F. (2007). Mitmachen oder zuhören? Mündliche Schülerinnen- und Schülerbeteiligung im Mathematikunterricht. Unterrichtswissenschaft, 35(2), 101-124.

Pauli, C., \& Reusser, K. (2000). Zur Rolle der Lehrperson beim kooperativen Lernen. Schweizerische Zeitschrift für Bildungswissenschaften, 22(3), 421-442.

Pauli, C., \& Reusser, K. (2006). Von international vergleichenden Video Surveys zur videobasierten Unterrichtsforschung und -entwicklung. Zeitschrift für Pädagogik, 52(6), 774-798.

Perrez, M., Huber, G.L., \& Geißler, K.A. (2006). 8 Psychologie der pädagogischen Interaktion. In A. Krapp \& B. Weidenmann (Hrsg.), Anwendung Psychologie. Pädagogische Psychologie: Ein Lehrbuch (5. Aufl. S. 357-421). Weinheim: Beltz.

van de Pol, J., Volman, M., \& Beishuizen, J. (2010). Scaffolding in teacher-student interaction: a decade of research. Educational Psychology Review, 22(3), 271-296. https://doi.org/10.1007/s10648-0109127-6.

Ranger, G. (2017). Kinder in kooperativen Lernphasen kognitiv aktivieren. GBV Gemeinsamer Bibliotheksverbund.

Renkl, A. (2011). Aktives Lernen: Von sinnvollen und weniger sinnvollen theoretischen Perspektiven zu einem schillernden Konstrukt. Unterrichtswissenschaft, 39(3), 197-212.

Ryu, S., \& Lombardi, D. (2015). Coding classroom interactions for collective and individual engagement. Educational Psychologist, 50(1), 70-83. https://doi.org/10.1080/00461520.2014.1001891.

Sedláček, M., \& Šed'ova, K. (2020). Are student engagement and peer relationships connected to student participation in classroom talk? Learning, Culture and Social Interaction, 26, 1-12. https://doi.org/ 10.1016/j.lcsi.2020.100411.

Seidel, T. (2014). Angebots-Nutzungs-Modelle in der Unterrichtspsychologie. Integration von Strukturund Prozessparadigma. Zeitschrift für Pädagogik, 60(6), 850-866.

Seidel, T. (2020). Kommentar zum Themenblock „Angebots-Nutzungs-Modelle als Rahmung“: Quo vadis deutsche Unterrichtsforschung? Modellierung von Angebot und Nutzung im Unterricht. Zeitschrift für Pädagogik, 66(Beiheft), 95-101.

Seidel, T., Dalehefte, I. M., \& Meyer, L. (2003). Aufzeichnen von Physikunterricht. In T. Seidel, M. Prenzel, R. Duit \& M. Lehrke (Hrsg.), IPN-Materialien. Technischer Bericht zur Videostudie „Lehr-LernProzesse im Physikunterricht" (S. 47-98). Kiel: IPN.

Steglich, C., \& Knecht, A. (2010). Die statistische Analyse dynamischer Netzwerke. In C. Stegbauer \& R. Häußling (Hrsg.), Handbuch Netzwerkforschung. Netzwerkforschung, (Bd. 4, S. 433-446). Wiesbaden: VS Verlag für Sozialwissenschaften, Springer Fachmedien.

Sulo, R., Berger-Wolf, T., \& Grossman, R. (2010). Meaningful selection of temporal resolution for dynamic networks. In U. Brefeld (Hrsg.), Proceedings of the Eighth Workshop on Mining and Learning with Graphs (S. 127-136). Washington, D.C.: ACM. https://doi.org/10.1145/1830252.1830269.

Troll, B., Pietsch, M., \& Besser, M. (2020). Verhaltensbezogenes engagement im Unterricht. Eine Analyse der Generalisierbarkeit und Zuverlässigkeit von Videobeobachtungen. Zeitschrift für Pädagogische Psychologie. https://doi.org/10.1024/1010-0652/a000286.

Vieluf, S., Praetorius, A.-K., Rakoczy, K., Kleinknecht, M., \& Pietsch, M. (2020). Angebots-Nutzungsmodelle der Wirkweise des Unterrichts: eine kritische Auseinandersetzung mit ihrer theoretischen Konzeption. Zeitschrift für Pädagogik, 66(Beiheft), 63-80.

Vygotsky, L.S. (Hrsg.). (1978). Mind in society: the development of higher psychological processes. Harvard University Press.

Wagner, C. J., \& González-Howard, M. (2018). Studying discourse as social interaction: the potential of social network analysis for discourse studies. Educational Researcher, 47(6), 375-383. https://doi. org/10.3102/0013189X18777741.

Wasserman, S., \& Faust, K. (Hrsg.). (1997). Social network analysis: methods and applications. Structural analysis in the social sciences. Bd. 8. Cambridge University Press.

Webb, N. M. (2009). The teacher's role in promoting collaborative dialogue in the classroom. The British journal of educational psychology, 79(1), 1-28. https://doi.org/10.1348/000709908X380772.

Zander, L., Kreutzmann, M., \& Hannover, B. (2017). Peerbeziehungen im Klassenzimmer. Zeitschrift für Erziehungswissenschaft, 20(3), 353-386. https://doi.org/10.1007/s11618-017-0768-9. 